\title{
Non-Gaussian disorder average in the Sachdev-Ye-Kitaev model
}

\author{
T. Krajewski, ${ }^{1, *}$ M. Laudonio, ${ }^{2,3, \dagger}$ R. Pascalie, ${ }^{2,4, \dagger}$ and A. Tanasa ${ }^{2,5, \S}$ \\ ${ }^{1}$ Aix Marseille University, Université de Toulon, CNRS, CPT, 13009 Marseille, France, EU \\ ${ }^{2}$ Université de Bordeaux, LaBRI, CNRS UMR 5800, 33405 Talence, France, EU \\ ${ }^{3}$ Department of Applied Mathematics, University of Waterloo, Waterloo, Ontario, Canada N2L $3 G 1$ \\ ${ }^{4}$ Mathematisches Institut der Westfalischen Wilhelms-Universitaat, 48149 Münster, Germany, EU \\ ${ }^{5}$ H. Hulubei National Institute of Physics and Nuclear Engineering, 077125 Magurele, \\ Romania, EU IUF, 75005 Paris, France, EU
}

(Received 21 December 2018; published 28 June 2019)

\begin{abstract}
We study the effect of the non-Gaussian average over the random couplings in a complex version of the celebrated Sachdev-Ye-Kitaev (SYK) model. Using a Polchinski-like equation and random tensor Gaussian universality, we show that the effect of this non-Gaussian averaging leads to a modification of the variance of the Gaussian distribution of couplings at leading order in $N$. We then derive the form of the effective action to all orders. An explicit computation of the modification of the variance in the case of a quartic perturbation is performed for both the complex SYK model mentioned above and the SYK generalization proposed in D. Gross and V. Rosenhaus [J. High Energy Phys. 02 (2017) 093].
\end{abstract}

DOI: 10.1103/PhysRevD.99.126014

\section{INTRODUCTION}

Quantum mechanical models involving $q$-body Hamiltonians were proposed in a nuclear physics setting in the 1970s (see the review article of Brody et al. [1] and references therein). Similarly, Hamiltonians involving simultaneous interactions between many particles were introduced by French and Wong in [2]. Many-particle eigenvalue densities, generated by such $q$-body interactions in asymptotically large spectroscopic spaces were studied by Mon and French in [3].

In the 1990s, in the context of condensed matter physics, the paper [4] of Sachdev and Ye then led to an important amount of interest. In a series of talks in 2015, Kitaev introduced a simplified version of this model and showed that it can be a particularly interesting toy model for AdS/ CFT physics. The model, called ever since the Sachdev-YeKitaev (SYK) model, has attracted a huge amount of interest for both condensed matter and high energy physics (see, for example, [5-11] or the review articles [12,13].

More specifically, the SYK model is a quantummechanical model with $N$ fermions with random

\footnotetext{
*thomas.krajewski@cpt.univ-mrs.fr

†matteo.laudonio@u-bordeaux.fr

romain.pascalie@u-bordeaux.fr

§ntanasa@u-bordeaux.fr
}

Published by the American Physical Society under the terms of the Creative Commons Attribution 4.0 International license. Further distribution of this work must maintain attribution to the author(s) and the published article's title, journal citation, and DOI. Funded by SCOAP. interactions involving $q$ of these fermions at a time. Each coupling $J$ is a variable drawn from a random Gaussian distribution. In this paper, we investigate the behavior of the model when this Gaussianity condition is relaxed.

We first work with a version of the SYK model containing $q$ flavors of complex fermions, each of them appearing once in the interaction. This model is very close in spirit to the colored tensor model (see the book [14]) and it is a particular case of a complex version of the GrossRosenhaus SYK generalization proposed in [8]. This particular version of the SYK model has already been studied in $[9,15-18]$.

Following the approach proposed in [19] for tensor models and group field theory (see also [20-22]), we first use a Polchinski-like flow equation to obtain Gaussian universality. This Gaussian universality result for the colored tensor model was initially proved in [23]. Let us also mention here that this universality result for colored tensor models was also exploited in [24], in a condensed matter physics setting, to identify an infinite universality class of infinite-range $p$-spin glasses with non-Gaussian correlated quenched distributions.

In this paper, we further obtain the effective action for the non-Gaussian averaged complex SYK model studied here. We show that the effect of this non-Gaussian averaging is a modification of the variance of the Gaussian distribution of couplings at leading order in $N$.

We then choose a specific quartic perturbation (known in the tensor model literature as a pillow or a melonic quartic perturbation, see, for example, [25-28] or the TASI lectures 
on large $N$ tensor models [29]) and, using the HubbardStratonovich (or the intermediate field representation) for the disorder $J$, we explicitly compute the first-order correction of the effective action and the modification of the Gaussian distribution of the couplings $J$ at leading order in $N$. We then generalize these explicit calculations for the Gross-Rosenhaus SYK model proposed in [8] (the fermionic fields being this time real) and, as above, we obtain the first-order correction of the Gross-Rosenhaus SYK effective action and the modification of the Gaussian distribution of the couplings $J$ at leading order in $N$.

For the sake of completeness, let us mention that, in [30], the four-point function of the SYK model in a doublescaling limit was computed and the random couplings did not necessarily have to be independent and Gaussian-it was enough for these random couplings to be taken as independent random variables, with zero mean and uniformly bounded moments independent of $N$.

Our paper is organized as follows. In the following section, we introduce the complex SYK model we initially work with and we express the non-Gaussian potential as a sum over particular graphs. In Sec. III, the Gaussian universality result is exhibited, using a Polchinski-like equation. Section IV is dedicated to the study of the effective action for this model. In Secs. V and VI, respectively, we perform our explicit calculations for quartic perturbations for the complex SYK and the (real) Gross-Rosenhaus SYK generalization. The last section lists some concluding remarks. For the sake of completeness, we add an Appendix that constructs the Dyson-Schwinger equations for the intermediate field used in this paper. This construction follows the lines of [31] and is done for both the complex SYK and the (real) Gross-Rosenhaus SYK generalization studied here.

\section{A COMPLEX SYK MODEL WITH NON-GAUSSIAN DISORDER}

As already announced in the previous section, we study here a complex SYK model with $q$ complex fermions $\psi_{i_{a}}^{a}(t)$, where the label $a=1, \ldots, q$ is the flavor and each fermion carries an index $i_{a}=1, \ldots, N$. The action writes

$$
S_{J}(\psi, \bar{\psi})=\int d t\left(\sum_{a, i_{a}} \bar{\psi}_{i_{a}}^{a} \partial_{t} \psi_{i_{a}}^{a}+\mathrm{i}^{\frac{q}{2}} \sum_{i_{1}, \ldots, i_{q}} \bar{J}_{i_{1}, \ldots, i_{q}} \psi_{i_{1}}^{1} \cdots \psi_{i_{q}}^{q}+\mathrm{i}^{\frac{q}{2}} \sum_{i_{1}, \ldots, i_{q}} J_{i_{1}, \ldots, i_{q}} \bar{\psi}_{i_{1}}^{1} \cdots \bar{\psi}_{i_{q}}^{q}\right) .
$$

Here $J_{i_{1}, \ldots, i_{q}}$ is a rank $q$ tensor that plays the role of a coupling constant. As already mentioned in the Introduction, this model is close in spirit to tensor models and is a particular case of the Gross-Rosenhaus generalization of the SYK model.

For the sake of completeness, let us mention that a bipartite complex SYK-like tensor model (without any fermion flavors) and with $O(N)^{3}$ symmetry was studied in the TASI lectures [29]. It was then found that one of the operators has a complex scaling dimension, which suggests that the nearly conformal large $N$ phase of the bipartite model is unstable.

The model (1) we study here is subject to quenched disorder-we average the free energy (or connected correlation functions) over the couplings $J$. The most convenient way to perform this is through the use of replicas. We thus add an extra replica index $r=1, \ldots, n$ to the fermions. One has

$$
\langle\log Z(J)\rangle_{J}=\lim _{n \rightarrow 0} \frac{\left\langle Z^{n}(J)\right\rangle_{J}-1}{n},
$$

with

$$
Z^{n}(J)=\int \prod_{1 \leq r \leq n}\left[d \psi_{r}\right]\left[d \bar{\psi}_{r}\right] \exp \sum_{r} S_{J}\left(\psi_{r}, \bar{\psi}_{r}\right) .
$$

The averaging over $J$ is performed with a possibly nonGaussian weight

$$
\left\langle Z^{n}(J)\right\rangle_{J}=\frac{\int d J d \bar{J} Z^{n}(J) \exp \left[-\left[\frac{N^{q-1}}{\sigma^{2}} J \bar{J}+V_{N}(J, \bar{J})\right]\right]}{\int d J d \bar{J} \exp \left[-\left[\frac{N^{q-1}}{\sigma^{2}} J \bar{J}+V_{N}(J, \bar{J})\right]\right]} .
$$

We further impose that the potential $V_{N}$ is invariant under independent unitary transformations,

$$
\begin{aligned}
J_{i_{1}, \ldots, i_{q}} & \rightarrow \sum_{j_{1}, \ldots, j_{q}} U_{i_{1} j_{1}}^{1} \cdots U_{i_{q} j_{q}}^{q} J_{j_{1}, \ldots, j_{q}}, \\
\bar{J}_{i_{1}, \ldots, i_{q}} & \rightarrow \sum_{j_{1}, \ldots, j_{q}} \bar{U}_{i_{1} j_{1}}^{1} \cdots \bar{U}_{i_{q} j_{q}}^{q} \bar{J}_{j_{1}, \ldots, j_{q}} .
\end{aligned}
$$

Assuming that the potential $V_{N}$ is a polynomial (or an analytic function) in the couplings $J$ and $\bar{J}$, this invariance imposes that the potential can be expanded over particular graphs, as follows. Let us consider (not necessarily connected) graphs ${ }^{1} \Gamma$ with black and white vertices of valence $q$. The edges of such a graph connect only black to white vertices (we thus have bipartite graphs) and are labeled by a color $a=1, \ldots, q$ in such a way that, at each vertex, the $q$ incident edges carry distinct colors (we thus have edgecolored graphs). Let us mention that each edge color of these graphs $\Gamma$ corresponds to a fermion flavor of the model. See Figs. 1(a) and 1(b) for some examples of such graphs, respectively, melonic and nonmelonic. A graph is

\footnotetext{
${ }^{1}$ These graphs are called bubbles in the tensor model literature (see again the book [14].)
} 

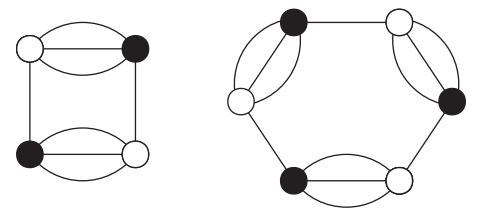

(a) Melonic graphs
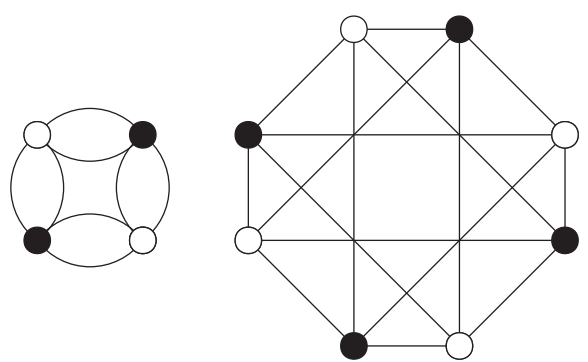

(b) Non-melonic graphs

FIG. 1. Examples of (a) melonic and (b) nonmelonic graphs for $q=4$.

called melonic if for any vertex $v$, there is another vertex $\bar{v}$ such that the removal of $v$ and $\bar{v}$ yields exactly $q$ connected components (including isolated lines).

The most general form of the potential is expanded over these graphs as

$$
V_{N}(J, \bar{J})=\sum_{\text {graph } \Gamma} \lambda_{\Gamma} \frac{N^{q-k(\Gamma)}}{\operatorname{sym}(\Gamma)}\langle J, \bar{J}\rangle_{\Gamma},
$$

where we have used the shorthand for the contraction of tensors along graph $\Gamma$

$$
\begin{aligned}
\langle J, \bar{J}\rangle_{\Gamma}= & \sum_{1 \leq i_{v, q}, \ldots, i_{\bar{v}, a} \leq N} \prod_{\substack{\text { white } \\
\text { vertices } v}} J_{i_{v, 1}, \ldots, i_{v, q}} \prod_{\substack{\text { black } \\
\text { vertices } \bar{v}}} \bar{J}_{\bar{i}_{\bar{v}, 1}, \ldots, \bar{i}_{\bar{v}, q}} \\
& \times \prod_{\substack{\text { edges } \\
e=(v, \bar{v})}} \delta_{i_{v, c}(e), i_{\bar{\nu}, c(e)}} \cdot
\end{aligned}
$$

In this expression, $\lambda_{\Gamma}$ is a real number, $k(\Gamma)$ is the number of connected components of $\Gamma$, and $\operatorname{sym}(\Gamma)$ is its symmetry factor. The contraction of indices means that each white vertex carries a tensor $J$ and each black vertex carries a tensor $\bar{J}$, and the indices have to be contracted by identifying two indices on both sides of an edge, the place of the index in the tensor being defined by the color of the edge denoted by $c(e)$.

The Gaussian term corresponds to a dipole graph (a white and a black vertex, related by $q$ lines) and reads

$$
\frac{N^{q-1}}{\sigma^{2}} J \bar{J}=\frac{N^{q-1}}{\sigma^{2}} \sum_{1 \leq i_{1}, \ldots, i_{q} \leq N} J_{i_{1}, \ldots, i_{q}} \bar{J}_{i_{1}, \ldots, i_{q}} .
$$

Introducing the pair of complex conjugate tensors $K$ and $\bar{K}$ defined by

$$
\begin{aligned}
& K_{i_{1}, \ldots, i_{q}}=\mathrm{i}^{\frac{q}{2}} \sum_{r} \int d t \psi_{i_{1}, r}^{1} \cdots \psi_{i_{q} ; r}^{q} \\
& \bar{K}_{i_{1}, \ldots, i_{q}}=\mathrm{i}^{\frac{q}{2}} \sum_{r} \int d t \bar{\psi}_{i_{1}, r}^{1} \cdots \bar{\psi}_{i_{q} ; r}^{q},
\end{aligned}
$$

the averaged partition function reads

$$
\left\langle Z^{n}(J)\right\rangle_{J}=\frac{\int[d \psi][d \bar{\psi}] \exp \left[-\int d t \sum_{a, i_{a}, r} \bar{\psi}_{i_{a}, r}^{a} \partial_{t} \psi_{i_{a}, r}^{a}\right] \int d J d \bar{J} \exp \left[-\left[\frac{N^{q-1}}{\sigma^{2}} J \bar{J}+V_{N}(J, \bar{J})+J \bar{K}+\bar{J} K\right]\right]}{\int d J d \bar{J} \exp \left[-\left[\frac{N^{q-1}}{\sigma^{2}} J \bar{J}+V_{N}(J, \bar{J})\right]\right]} .
$$

After a shift of variables in the integral over $J$ and $\bar{J}$, the integral on $J$ and $\bar{J}$ in the numerator reads

$$
\exp \left[-\frac{\sigma^{2}}{N^{q-1}} K \bar{K}\right] \int d J d \bar{J} \exp -\left[\frac{N^{q-1}}{\sigma^{2}} J \bar{J}+V_{N}\left(J-\frac{\sigma^{2}}{N^{q-1}} K, \bar{J}-\frac{\sigma^{2}}{N^{q-1}} \bar{K}\right)\right] .
$$

In order to study the large $N$ limit of the average (10), we introduce the background field effective potential, with $L=-\frac{\sigma^{2}}{N^{q-1}} K$ and $\bar{L}=-\frac{\sigma^{2}}{N^{q-1}} \bar{K}$. One has

$$
V_{N}(s, L, \bar{L})=-\log \int d J d \bar{J} \exp -\left[\frac{N^{q-1}}{s} J \bar{J}+V_{N}(J+L, \bar{J}+\bar{L})\right]+N^{q} \log \frac{\pi s}{N^{q-1}} .
$$

In this framework, $s$ is a parameter that interpolates between the integral we have to compute, at $s=\sigma^{2}$ (up to a trivial multiplicative constant), and the potential we started with at $s=0$ (no integration and $J=\bar{J}=0$ ). The inclusion of the constant ensures that the effective potential remains zero when we start with a vanishing potential. This comes to 


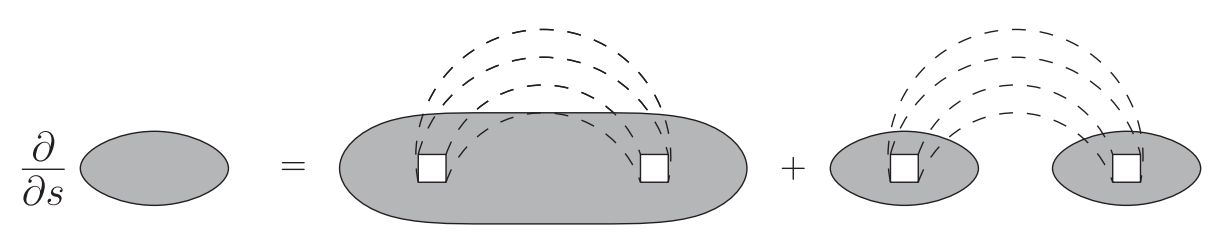

FIG. 2. Graphical representation of Eq. (14) for $q=4$.

$$
\begin{aligned}
& \int d J d \bar{J} \exp \left[-\left[\frac{N^{q-1}}{\sigma^{2}} J \bar{J}+V_{N}\left(J-\frac{\sigma^{2}}{N^{q-1}} K, \bar{J}-\frac{\sigma^{2}}{N^{q-1}} \bar{K}\right)\right]\right] \\
& =\left(\frac{N^{q-1}}{\pi s}\right)^{N^{q}} \exp \left[-V_{N}\left(s=\sigma^{2}, L=-\frac{\sigma^{2}}{N^{q-1}} K, \bar{L}=-\frac{\sigma^{2}}{N^{q-1}} \bar{K}\right)\right] .
\end{aligned}
$$

In the next section, we will derive the large $N$ behavior of the effective potential using a Polchinski-like flow equation.

\section{GAUSSIAN UNIVERSALITY}

As already mentioned in the Introduction, this section follows the approach proposed in [19] (see also [20-22]). Using standard quantum field theoretical manipulations (see, for example, the book [32]), one can show that the effective potential $V_{N}(s, L, \bar{L})$ [see Eq. (12)] obeys the following differential equation:

$$
\begin{aligned}
\frac{\partial V}{\partial s}= & \frac{1}{N^{q-1}} \sum_{1 \leq i_{1}, \ldots, i_{q} \leq N}\left(\frac{\partial^{2} V}{\partial L_{i_{1}, \ldots, i_{q}} \partial \bar{L}_{i_{1}, \ldots, i_{q}}}\right. \\
& \left.-\frac{\partial V}{\partial L_{i_{1}, \ldots, i_{q}}} \frac{\partial V}{\partial \bar{L}_{i_{1}, \ldots, i_{q}}}\right) .
\end{aligned}
$$

One can represent this equation in a graphical way as shown in Fig. 2. The first term on the rhs corresponds to an edge closing a loop in the graph and the second term on the rhs corresponds to a bridge (or a one-particle reducible) edge. This equation is formally a Polchinski-like equation [33], albeit there are no short distance degrees of freedom over which we integrate. In our context, it simply describes a partial integration with a weight $s$ and will be used to control the large $N$ limit of the effective potential.

Since the effective potential is also invariant under the unitary transformations defined in Eq. (5), it may also be

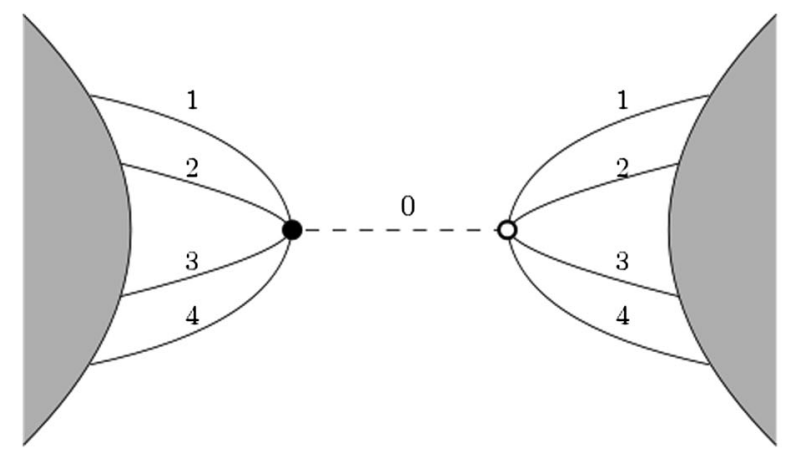

expanded over graphs as in (7),

$$
V_{N}(s, L, \bar{L})=\sum_{\text {graph } \Gamma} \lambda_{\Gamma}(s) \frac{N^{q-k(\Gamma)}}{\operatorname{sym}(\Gamma)}\langle L, \bar{L}\rangle_{\Gamma},
$$

with $s$ dependent couplings $\lambda_{\Gamma}(s)$.

Inserting this graphical expansion in the differential equation (14), we obtain a system of differential equations for the couplings,

$\frac{d \lambda_{\Gamma}}{d s}=\sum_{\Gamma^{\prime} /(\bar{v} v)=\Gamma} N^{k(\Gamma)-k\left(\Gamma^{\prime}\right)+e(v, \bar{v})-q+1} \lambda_{\Gamma^{\prime}}-\sum_{\left(\Gamma^{\prime} \cup \Gamma^{\prime \prime}\right) /(\bar{v} v)=\Gamma} \lambda_{\Gamma^{\prime}} \lambda_{\Gamma^{\prime \prime}}$.

A derivation of the potential $V$ with respect to $L_{i_{1}, \ldots, i_{q}}$ (respectively, $\bar{L}_{i_{1}, \ldots, i_{q}}$ ) removes a white vertex (respectively, a black vertex). Then, the summation over the indices in $i_{1}, \ldots, i_{q}$ in (14) reconnects the edges, respecting the colors.

In the first term on the rhs of (14), given a graph $\Gamma$ in the expansion of the lhs, we have to sum over all graphs $\Gamma^{\prime}$ and pairs of a white vertex $v$ and a black vertex $\bar{v}$ in $\Gamma^{\prime}$ such that the graph $\Gamma^{\prime} /(\bar{v} v)$ obtained after reconnecting the edges (discarding the connected components made of single lines) is equal to $\Gamma$ (see Figs. 3 and 4).

The number $e(v, \bar{v})$ is the number of edges directly connecting $v$ and $\bar{v}$ in $\Gamma$. After summation over the indices,

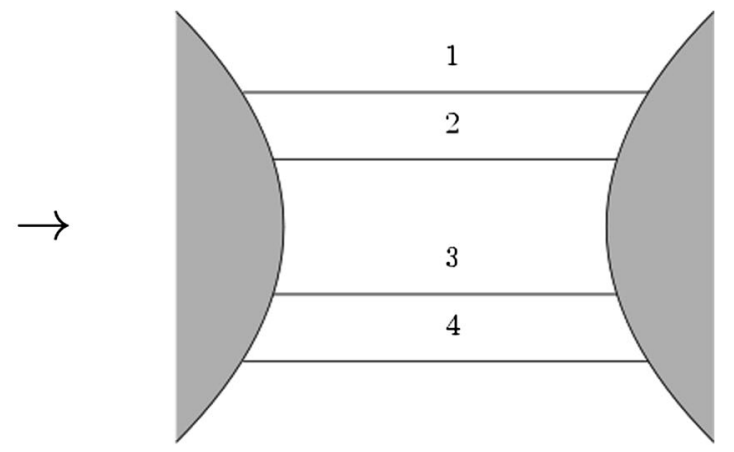

FIG. 3. Removal of a white and a black vertex and reconnection of the edges. 

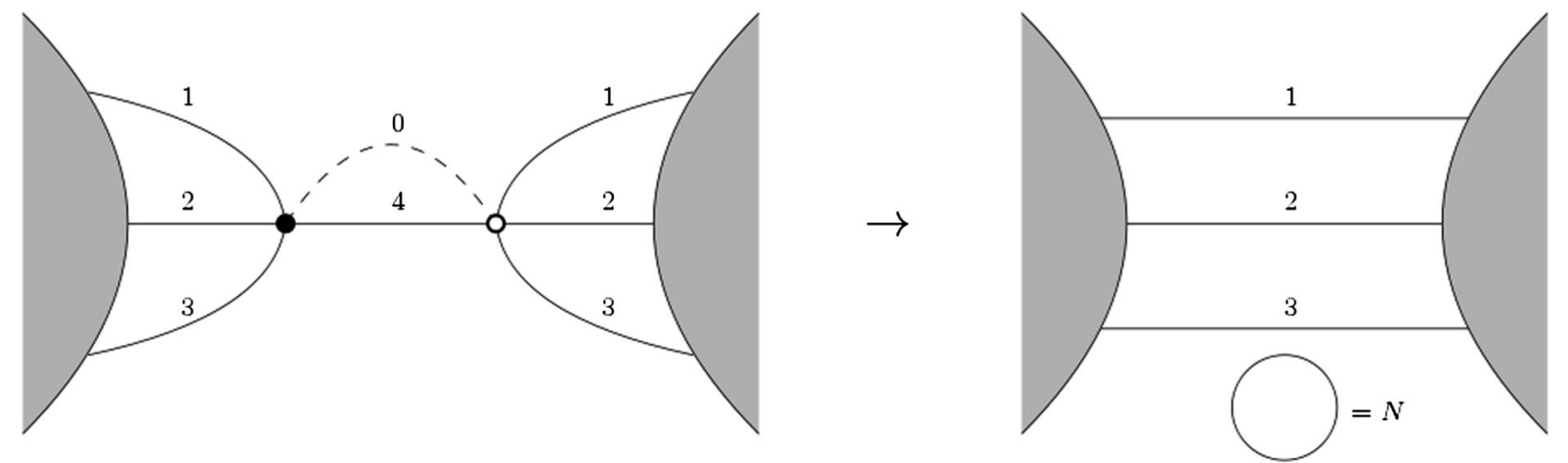

FIG. 4. Removal of a white and a black vertex and reconnection of the edges creating a loop.

each of these lines yields a power of $N$, which gives the factor of $N^{e(v, \bar{v})}$.

The operation of removing two vertices and reconnecting the edges can at most increase the number of connected components (including the graphs made of single closed lines) by $q-1$, so that we always have $k(\Gamma)-k\left(\Gamma^{\prime}\right)+e(v, \bar{v})-q+1 \leq 0$. We obtain the equality if and only if $\Gamma^{\prime}$ is a melonic graph. Therefore, in the large $N$ limit, only melonic graphs survive in the first term on the rhs of (16).

In the second term, we sum over graphs $\Gamma^{\prime}$ and white vertices $v \in \Gamma^{\prime}$ and graphs $\Gamma^{\prime \prime}$ and black vertices $\bar{v} \in \Gamma^{\prime \prime}$, with the condition that the graph obtained after removing the vertices and reconnecting the lines $\left(\Gamma^{\prime} \cup \Gamma^{\prime \prime}\right) /(\bar{v} v)$ is equal to $\Gamma$. In that case, the number of connected components necessarily diminishes by one, so that all powers of $N$ cancel.

The crucial point in the system (16) is that only negative (or null) powers of $N$ appear. It can be written as

$$
\frac{d \lambda_{\Gamma}}{d s}=\beta_{0}\left(\left\{\lambda_{\Gamma}\right\}\right)+\frac{1}{N} \beta_{1}\left(\left\{\lambda_{\Gamma}\right\}\right)+\cdots
$$

As a consequence, if $\lambda_{\Gamma}(s=0)$ is bounded, then $\lambda_{\Gamma}(s)$ is also bounded for all $s$ (i.e., it does not contain positive powers of $N$ ).

Let us now substitute $L=-\frac{\sigma^{2}}{N^{q-1}} K$ and $\bar{L}=-\frac{\sigma^{2}}{N^{q-1}} \bar{K}$ in the expansion of the effective potential (7),

$$
\begin{aligned}
& V_{N}\left(s=\sigma^{2}, L=-\frac{\sigma^{2}}{N^{q-1}} K, \bar{L}=-\frac{\sigma^{2}}{N^{q-1}} \bar{K}\right) \\
& \quad=\sum_{\operatorname{graph} \Gamma} \lambda_{\Gamma}\left(\sigma^{2}\right) \frac{\left(-\sigma^{2}\right)^{v(\Gamma)} N^{q-k(q)-(q-1) v(\Gamma)}}{\operatorname{sym}(\Gamma)}\langle K, \bar{K}\rangle_{\Gamma} .
\end{aligned}
$$

Here $v(\Gamma)$ is the number of vertices of $\Gamma$. The exponent of $N$ can be rewritten as $(q-1)(1-v(\Gamma))+1-k(\Gamma)$. It has a maximal value for $v(\Gamma)=2$ and $k(\Gamma)=1$, which corresponds to the dipole graph. This is a reexpression of the Gaussian universality property of random tensors.

\section{EFFECTIVE ACTION}

Taking into account the non-Gaussian quenched disorder, we derive the effective action for the bilocal invariants,

$$
G_{r, r^{\prime}}^{a}\left(t, t^{\prime}\right)=\frac{1}{N} \sum_{i} \psi_{i, r}^{a}\left(t_{1}\right) \bar{\psi}_{i, r^{\prime}}^{a}\left(t^{\prime}\right)
$$

Note that these invariants carry one flavor label $a$ and two replica indices $r, r^{\prime}$.

To this end, let us come back to the partition function (10). We then express the result of the average over $J$ and $\bar{J}$ as a sum over graphs $\Gamma$ using the expansion of the effective potential (18) and replacing the tensors $K$ and $\bar{K}$ in terms of the fermions $\psi$ and $\bar{\psi}$ [see Eq. (9)].

Each graph $\Gamma$ then involves the combination

$$
\begin{aligned}
\langle K, \bar{K}\rangle_{\Gamma}= & \sum_{1 \leq i_{v, a}, \ldots, i_{\bar{v}, a} \leq N} \prod_{\substack{\text { white } \\
\text { vertices } v}} \sum_{r_{v}} \int d t_{v} \psi_{i_{v, 1}, r_{v}}^{1}\left(t_{v}\right) \cdots \\
& \times \psi_{i_{v, q}, r_{v}}^{q}\left(t_{v}\right) \prod_{\substack{\text { black } \\
\text { vertices } \bar{v}}} \sum_{r_{\bar{v}}} \int d t_{\bar{v}} \bar{\psi}_{\bar{i}_{\bar{v}, 1}, r_{\bar{v}}}^{1} \ldots \\
& \times \bar{\psi}_{\bar{i}_{\bar{v}, q}, \bar{v}}^{q}\left(t_{\bar{v}}\right) \prod_{\substack{\text { edges } \\
e=(v, \bar{v})}} \delta_{i_{v, c(e)}, i_{\bar{v}, c(e)}} .
\end{aligned}
$$

After introducing the Lagrange multiplier $\Sigma$ to enforce the constraint (19) and assuming a replica symmetric saddle point, the effective action of our model writes

$$
\begin{aligned}
\frac{\mathcal{S}_{\mathrm{eff}}[\mathrm{G}, \Sigma]}{N}= & -\sum_{f=1}^{q} \log \operatorname{det}\left(\delta\left(t_{1}-t_{2}\right) \partial_{t}-\Sigma_{f}\left(t_{1}, t_{2}\right)\right) \\
& +\int \mathrm{d} \mathbf{t} \sum_{f=1}^{4} \Sigma_{f}(\mathbf{t}) G_{f}(\mathbf{t}) \\
& -\sum_{\Gamma} N^{-(v(\Gamma)-2)(q / 2-1)+1-k(\Gamma)} \mu_{\Gamma}\left(\sigma^{2},\left\{\lambda_{\Gamma^{\prime}}\right\}\right)\langle G\rangle_{\Gamma} .
\end{aligned}
$$

The term $\langle G\rangle_{\Gamma}$ associated with a graph $\Gamma$ is constructed as follows:

(1) To each vertex associate a real variable $t_{v}$.

(2) To an edge of color $c$ joining $v$ to $v^{\prime}$ associate $G_{c}\left(t_{v}, t_{v^{\prime}}\right)$. 
(3) Multiply all edge contributions and integrate over vertex variables.

See Fig. 5 for a graphical representation of the term $<$ $G>_{\Gamma}$ for the quartic melonic graph for $q=4$. We then add up these contributions, with a weight $\lambda_{\Gamma}$ and a power of $N$ given by [with $e(\gamma)$ the number of edges of $\Gamma$, obeying $2 e(\Gamma)=q v(\gamma)]$

$$
\begin{aligned}
& N^{q-k(\Gamma)} \times\left(N^{-(q-1)}\right)^{v(\Gamma)} \times N^{e(\Gamma)} \\
& \quad=N \times N^{-(v(\Gamma)-2)(q / 2-1)+1-k(\Gamma) .}
\end{aligned}
$$

At leading order in $N$, only the Gaussian terms survive [i.e., the graph $\Gamma$ with $(v(\Gamma)=2$ and $k(\Gamma)=1)$ ], except for the matrix model case $(q=2)$. In this case, all terms corresponding to connected graphs survive. Let us emphasize that the variance of the Gaussian distribution of coupling is thus modified, as a consequence of the nonGaussian averaging of our model. Remarkably, for $q>2$, this is the only modification at leading order in $N$.

Moreover, the actual value of the covariance (which we denote by $\sigma^{\prime}$ ) induced by non-Gaussian disorder is most easily computed using a Schwinger-Dyson equation (see [34]). In our context, the latter arises from

$$
\begin{aligned}
& \sum_{i_{1} \ldots i_{q}} \int d J d \bar{J} \frac{\partial}{\partial \bar{J}_{i_{1} \ldots i_{q}}}\left\{J _ { i _ { 1 } \ldots i _ { q } } \operatorname { e x p } \left[-\left[\frac{N^{q-1}}{\sigma^{2}} J \bar{J}\right.\right.\right. \\
& \left.\left.\left.+V_{N}(J, \bar{J})\right]\right]\right\}=0 .
\end{aligned}
$$

At large $N$, it leads to the algebraic equation

$$
1=\frac{\sigma^{\prime 2}}{\sigma^{2}}+\sum_{\text {melonic graph } \Gamma} \frac{\lambda_{\Gamma}}{\operatorname{sym}(\Gamma)}\left(\sigma^{\prime}\right)^{v(\Gamma)} .
$$

Let us end this section by emphasizing that the new terms induced by non-Gaussian randomness do not lead to any terms that could break reparametrization invariance in situations where the kinetic term can be omitted.

This effective action, despite being nonlocal, is invariant under reparametrization (in the IR) at all orders in $1 / \mathrm{N}$,

$$
G\left(t, t^{\prime}\right) \rightarrow\left(\frac{d \phi}{d t}(t)\right)^{\Delta}\left(\frac{d \phi}{d t^{\prime}}\left(t^{\prime}\right)\right)^{\Delta} G\left(\phi(t), \phi\left(t^{\prime}\right)\right) .
$$

Indeed, changing the vertex variables as $t_{v} \rightarrow \phi\left(t_{v}\right)$, the Jacobians exactly cancel with the rescaling of $G$ since $\Delta=1 / q$ and all vertices are $q$ valent.

\section{A QUARTIC PERTURBATION COMPUTATION}

In this section, we consider the case $q=4$ with a quartic perturbation of the disorder. We explicitly compute the modification of the variance with respect to the Gaussian averaged model and we write down the effective action.

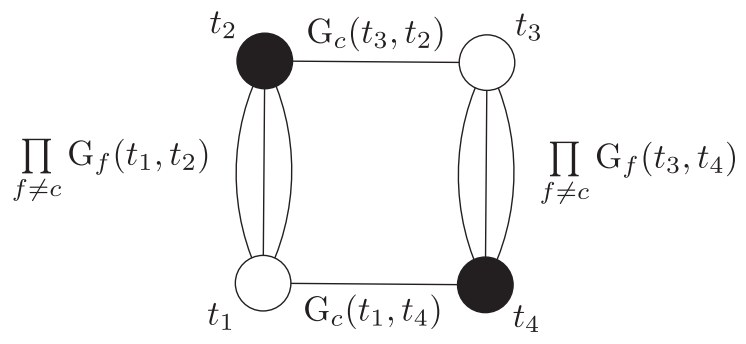

FIG. 5. Graphical representation of the term $\langle G\rangle_{\Gamma}$ for the quartic melonic graph for $q=4$.

\section{A. The quartic perturbed model}

The action writes

$$
\begin{aligned}
\mathcal{S}[\psi, \bar{\psi}]= & \int \mathrm{d} t\left(\sum_{f=1}^{4} \sum_{i=1} \bar{\psi}_{i}^{f} \frac{\mathrm{d}}{\mathrm{d} t} \psi_{i}^{f}-\sum_{i, j, k, l} \bar{J}_{i j k l} \psi_{i}^{1} \psi_{j}^{2} \psi_{k}^{3} \psi_{l}^{4}\right. \\
& \left.-\sum_{i, j, k, l} J_{i j k l} \bar{\psi}_{i}^{1} \bar{\psi}_{j}^{2} \bar{\psi}_{k}^{3} \bar{\psi}_{l}^{4}\right) .
\end{aligned}
$$

The coupling constant is a random tensor of rank 4 with the non-Gaussian potential given by

$$
V_{N}(J, \bar{J})=N^{3} \lambda \sum_{c=1}^{4} \sum_{I, K} J_{I} \bar{J}_{I_{\hat{c}} k_{c}} J_{K} \bar{J}_{K_{\hat{c}} i_{c}},
$$

where $I=\left(i_{1}, i_{2}, i_{3}, i_{4}\right)$, and $I_{\hat{c}} k_{c}$ means that $i_{c}$ is replaced by $k_{c}$. In the tensor model literature, this quartic term is called the melonic quartic term or the pillow term (see, for example, [25-28] or the TASI lectures on large $N$ tensor models [29]; see Fig. 6).

We need to integrate over the disorder the replicated generating functional

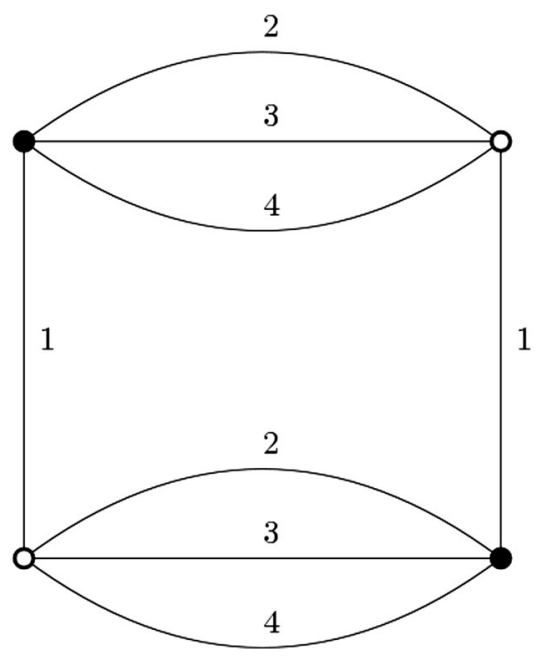

FIG. 6. The pillow term for a particular choice of colors of its edges. 


$$
\begin{aligned}
\left\langle\mathrm{Z}^{n}\right\rangle_{J}= & \int \mathcal{D} \psi_{i}^{f, a} \mathcal{D} \bar{\psi}_{i}^{f, a} \mathcal{D} J \mathcal{D} \bar{J} \exp \left(-\int \mathrm{d} t\left(\sum_{a=1}^{n} \sum_{f=1}^{4} \sum_{i} \bar{\psi}_{i}^{f, a} \frac{\mathrm{d}}{\mathrm{d} t} \psi_{i}^{f, a}\right)-\frac{N^{3}}{\sigma^{2}} \sum_{i, j, k, l} J_{i j k l} \bar{J}_{i j k l}\right. \\
& \left.+\int \mathrm{d} t\left(\sum_{a=1}^{n} \sum_{i, j, k, l} \bar{J}_{i j k l} \psi_{i}^{1, a} \psi_{j}^{2, a} \psi_{k}^{3, a} \psi_{l}^{4, a}+\sum_{a=1}^{n} \sum_{i, j, k, l} J_{i j k l} \bar{\psi}_{i}^{1, a} \bar{\psi}_{j}^{2, a} \bar{\psi}_{k}^{3, a} \bar{\psi}_{l}^{4, a}\right)-V_{N}(J, \bar{J})\right) .
\end{aligned}
$$

\section{B. Hubbard-Stratonovich transformation for the disorder}

We start by rewriting the quartic term in $J$ and $\bar{J}$ using a Hubbard-Stratonovich transformation (or intermediate field representation, see, for example, [31,35])

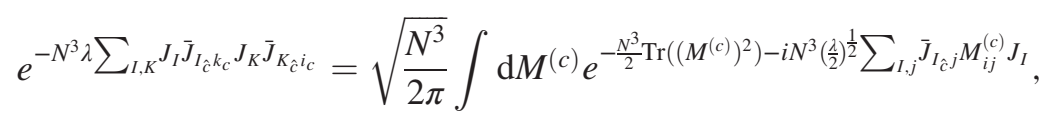

where $M^{(c)}$ is an $N \times N$ Hermitian matrix, for $c \in\{1,2,3,4\}$. In (28), keeping only the terms in $J$ and $\bar{J}$, we get

$$
\begin{gathered}
\int \mathcal{D} J \mathcal{D} \bar{J} \exp \left(-\frac{N^{3}}{\sigma^{2}} \sum_{i, j, k, l} J_{i j k l} \bar{J}_{i j k l}-i N^{3}\left(\frac{\lambda}{2}\right)^{\frac{1}{2}} \sum_{c=1}^{4} \sum_{I, j} J_{I} M_{i j}^{(c)} \bar{J}_{I_{\hat{c}} j}\right. \\
\left.+\sum_{a=1}^{n} \sum_{i, j, k, l}\left(\bar{J}_{i j k l} \psi_{i}^{1, a} \psi_{j}^{2, a} \psi_{k}^{3, a} \psi_{l}^{4, a}+J_{i j k l} \bar{\psi}_{i}^{1, a} \bar{\psi}_{j}^{2, a} \bar{\psi}_{k}^{3, a} \bar{\psi}_{l}^{4, a}\right)\right)
\end{gathered}
$$

Following [31], we introduce the notation $\mathcal{M}_{c}=\mathbb{1}^{\otimes(c-1)} \otimes M^{(c)} \otimes \mathbb{1}^{\otimes(4-c)}$ for $c \in\{1,2,3,4\}$. We can thus rewrite Eq. (30) as

$$
\int \mathcal{D} J \mathcal{D} \bar{J} \exp \left(-N^{3} \bar{J}\left(\frac{1}{\sigma^{2}} \mathbb{1}^{\otimes 4}+i\left(\frac{\lambda}{2}\right)^{\frac{1}{2}} \sum_{c=1}^{4} \mathcal{M}_{c}\right) J+\sum_{a=1}^{n} \sum_{i, j, k, l}\left(\bar{J}_{i j k l} \psi_{i}^{1, a} \psi_{j}^{2, a} \psi_{k}^{3, a} \psi_{l}^{4, a}+J_{i j k l} \bar{\psi}_{i}^{1, a} \bar{\psi}_{j}^{2, a} \bar{\psi}_{k}^{3, a} \bar{\psi}_{l}^{4, a}\right)\right) .
$$

Then, after rescaling $(J, \bar{J}) \rightarrow N^{\frac{3}{2}}(J, \bar{J})$, we can perform the integral over the disorder. Equation (31) thus rewrites

$$
\frac{(2 \pi)^{N}}{\operatorname{det}\left(\frac{1}{\sigma^{2}} \mathbb{1}^{\otimes 4}+i\left(\frac{\lambda}{2}\right)^{\frac{1}{2}} \sum_{c=1}^{4} \mathcal{M}_{c}\right)} \exp \left(N^{-3} \int \mathrm{d} t_{1} \mathrm{~d} t_{2} \sum_{a, b=1}^{n} \prod_{f=1}^{4} \psi^{f, a}\left(t_{1}\right)\left(\frac{1}{\sigma^{2}} \mathbb{1}^{\otimes 4}+i\left(\frac{\lambda}{2}\right)^{\frac{1}{2}} \sum_{c=1}^{4} \mathcal{M}_{c}\right)^{-1} \prod_{f=1}^{4} \bar{\psi}^{f, b}\left(t_{2}\right)\right) .
$$

Hence, the replicated generating functional (28) writes

$$
\begin{aligned}
\left\langle\mathrm{Z}^{n}\right\rangle_{J}= & \int \mathcal{D} \psi_{i}^{f, a} \mathcal{D} \bar{\psi}_{i}^{f, a} \mathcal{D} M^{(c)} \exp \left(-\int \mathrm{d} t \sum_{a=1}^{n} \sum_{f=1}^{4} \sum_{i} \bar{\psi}_{i}^{f, a} \frac{\mathrm{d}}{\mathrm{d} t} \psi_{i}^{f, a}-\operatorname{Tr} \log \left(\frac{1}{\sigma^{2}} \mathbb{1}^{\otimes 4}+i\left(\frac{\lambda}{2}\right)^{\frac{1}{2}} \sum_{c=1}^{4} \mathcal{M}_{c}\right)\right. \\
& \left.-\frac{1}{2} \sum_{c=1}^{4} \operatorname{Tr}\left(\mathcal{M}_{c}^{2}\right)+\frac{1}{N^{3}} \int \mathrm{d} t_{1} \mathrm{~d} t_{2} \sum_{a, b=1}^{n} \psi^{1, a} \psi^{2, a} \psi^{3, a} \psi^{4, a}\left(\frac{1}{\sigma^{2}} \mathbb{1}^{\otimes 4}+i\left(\frac{\lambda}{2}\right)^{\frac{1}{2}} \sum_{c=1}^{4} \mathcal{M}_{c}\right)^{-1} \bar{\psi}^{1, b} \bar{\psi}^{2, b} \bar{\psi}^{3, b} \bar{\psi}^{4, b}\right) .
\end{aligned}
$$

One has

$$
\begin{aligned}
\left(\frac{1}{\sigma^{2}} \rrbracket^{\otimes 4}+i\left(\frac{\lambda}{2}\right)^{\frac{1}{2}} \sum_{c=1}^{4} \mathcal{M}_{c}\right)^{-1} & =\sigma^{2} \rrbracket^{\otimes 4}+\sigma^{2} \sum_{k \geq 1} \frac{\left(-i\left(\frac{\lambda}{2}\right)^{\frac{1}{2}} \sigma^{2}\right)^{k}}{k !}\left(\sum_{c=1}^{4} \mathcal{M}_{c}\right)^{k} \\
& =\sigma^{2} \rrbracket^{\otimes 4}+\sigma^{2} \sum_{k \geq 1} \frac{\left(-i\left(\frac{\lambda}{2}\right)^{\frac{1}{2}} \sigma^{2}\right)^{k}}{k !} \sum_{k_{1}+k_{2}+k_{3}+k_{4}=k}\left(\begin{array}{c}
k \\
k_{1}, k_{2}, k_{3}, k_{4}
\end{array}\right) \prod_{c=1}^{4} \mathcal{M}_{c}^{k_{c}},
\end{aligned}
$$




$$
\begin{aligned}
\log \left(\frac{1}{\sigma^{2}} \mathbb{1}^{\otimes 4}+i\left(\frac{\lambda}{2}\right)^{\frac{1}{2}} \sum_{c=1}^{4} \mathcal{M}_{c}\right) & =-2 \log \left(\sigma \mathbb{1}^{\otimes 4}\right)+\log \left(\mathbb{1}^{\otimes 4}+i \sigma^{2}\left(\frac{\lambda}{2}\right)^{\frac{1}{2}} \sum_{c=1}^{4} \mathcal{M}_{c}\right) \\
& =-2 \log \left(\sigma \mathbb{1}^{\otimes 4}\right)-\sum_{k \geq 1} \frac{\left(-i\left(\frac{\lambda}{2}\right)^{\frac{1}{2}} \sigma^{2}\right)^{k}}{k} \sum_{k_{1}+k_{2}+k_{3}+k_{4}=k}\left(\begin{array}{c}
k \\
k_{1}, k_{2}, k_{3}, k_{4}
\end{array}\right) \prod_{c=1}^{4} \mathcal{M}_{c}^{k_{c}} .
\end{aligned}
$$

Inserting these series in (33) and keeping only the terms in $\mathcal{M}_{c}$, we have

$$
\begin{aligned}
- & \frac{1}{2} \sum_{c=1}^{4} \operatorname{Tr}\left(\mathcal{M}_{c}^{2}\right)+\sum_{k \geq 1} \frac{\left(-i\left(\frac{\lambda}{2}\right)^{\frac{1}{2}} \sigma^{2}\right)^{k}}{k} \sum_{k_{1}+k_{2}+k_{3}+k_{4}=k}\left(\begin{array}{c}
k \\
k_{1}, k_{2}, k_{3}, k_{4}
\end{array}\right) \operatorname{Tr}\left(\prod_{c=1}^{4} \mathcal{M}_{c}^{k_{c}}\right) \\
& +\frac{\sigma^{2}}{N^{3}} \int \mathrm{d} t_{1} \mathrm{~d} t_{2} \sum_{a, b=1}^{n} \psi^{1, a} \psi^{2, a} \psi^{3, a} \psi^{4, a} \sum_{k \geq 1} \frac{\left(-i\left(\frac{\lambda}{2}\right)^{\frac{1}{2}} \sigma^{2}\right)^{k}}{k !} \sum_{k_{1}+k_{2}+k_{3}+k_{4}=k}\left(\begin{array}{c}
k \\
k_{1}, k_{2}, k_{3}, k_{4}
\end{array}\right) \prod_{c=1}^{4} \mathcal{M}_{c}^{k_{c}} \bar{\psi}^{1, b} \bar{\psi}^{2, b} \bar{\psi}^{3, b} \bar{\psi}^{4, b} .
\end{aligned}
$$

Equation (36) can be rewritten in the form

$$
-\frac{1}{2} \sum_{c=1}^{4} \operatorname{Tr}\left(\mathcal{M}_{c}^{2}\right)+\operatorname{Tr}\left(\sum_{k \geq 1} \sum_{k_{1}+k_{2}+k_{3}+k_{4}=k} \frac{\left(-i\left(\frac{\lambda}{2}\right)^{\frac{1}{2}} \sigma^{2}\right)^{k}}{k_{1} ! k_{2} ! k_{3} ! k_{4} !}\left((k-1) ! \rrbracket^{\otimes 4}+\frac{\sigma^{2}}{N^{3}} \int \mathrm{d} \mathbf{t} \sum_{a, b=1}^{n}\left(\bar{\psi}_{b}^{4} \cdot \psi_{a}^{4}\right)\right) \prod_{c=1}^{4} \mathcal{M}_{c}^{k_{c}}\right)
$$

where $\left(\bar{\psi}_{b}^{4} \cdot \psi_{a}^{4}\right)=\bigotimes_{f=1}^{4}\left(\bar{\psi}^{f, b} \cdot \psi^{f, a}\right)$ with $\left(\bar{\psi}^{f, b} \cdot \psi^{f, a}\right)_{i j}=\bar{\psi}_{i}^{f, b} \psi_{j}^{f, a}$ and $\mathrm{dt}=\mathrm{d} t_{1} \mathrm{~d} t_{2}$.

We can note that the term proportional to the identity in (34) contributes to the effective action as the Gaussian part of the disorder.

\section{First-order correction of the effective action}

The integration on the intermediate fields $M^{(c)}$ cannot be explicitly performed. We can, however, truncate the series in $\lambda$ and compute perturbatively the first order of the effective action. Keeping only the linear and quadratic terms in the intermediate fields in Eq. (37), we get

$$
-\frac{1}{2} \sum_{c=1}^{4} \operatorname{Tr}\left(\mathcal{M}_{c}^{2}\right)-i \sigma^{2}\left(\frac{\lambda}{2}\right)^{\frac{1}{2}} \sum_{c=1}^{4} \operatorname{Tr}\left(\mathcal{M}_{c}\right)+\frac{\sigma^{2}}{N^{3}} \int \mathrm{d} t_{1} \mathrm{~d} t_{2} \sum_{a, b=1}^{n} \psi_{a}^{4}\left(-i\left(\frac{\lambda}{2}\right)^{\frac{1}{2}} \sigma^{2} \sum_{c=1}^{4} \mathcal{M}_{c}\right) \bar{\psi}_{b}^{4},
$$

where we have introduced the notations $\psi_{a}^{4}=\psi^{1, a} \psi^{2, a} \psi^{3, a} \psi^{4, a}$ and $\bar{\psi}_{b}^{4}=\bar{\psi}^{1, b} \bar{\psi}^{2, b} \bar{\psi}^{3, b} \bar{\psi}^{4, b}$. By performing partial traces on the identity part of $\mathcal{M}_{c}$, Eq. (38) can be further simplified,

$$
\int \prod_{c=1}^{4} \mathrm{~d} M^{(c)} \exp \left\{-\frac{N^{3}}{2} \sum_{c=1}^{4} \operatorname{Tr}\left(\left(M^{(c)}\right)^{2}\right)-\sum_{c=1}^{4} \operatorname{Tr}\left[\left(i\left(\frac{\lambda}{2}\right)^{\frac{1}{2}}\left(N^{3} \sigma^{2} \mathbb{1}+\frac{\sigma^{4}}{N^{3}} \int \mathrm{d} \mathbf{t} \sum_{a, b=1}^{n}\left(\bar{\psi}_{b}^{4} \cdot \psi_{a}^{4}\right)_{c}\right) M^{(c)}\right]\right\}\right.
$$

where $\left(\bar{\psi}_{b}^{4} \cdot \psi_{a}^{4}\right)_{c}=\prod_{d \neq c} \operatorname{Tr}\left(\bar{\psi}^{d, b} \cdot \psi^{d, a}\right)\left(\bar{\psi}^{c, b} \cdot \psi^{c, a}\right)$. Let us now perform the Gaussian integrals on the intermediate fields. We get

$$
\begin{aligned}
& \exp \left(-\frac{N^{3} \lambda}{4} \sum_{c=1}^{4} \operatorname{Tr}\left(\sigma^{2} \mathbb{1}+\frac{\sigma^{4}}{N^{6}} \int \mathrm{d} \mathbf{t} \sum_{a, b=1}^{n}\left(\bar{\psi}_{b}^{4} \cdot \psi_{a}^{4}\right)_{c}\right)^{2}\right) \\
& \left.=\exp \left(-N^{4} \lambda \sigma^{4}-\frac{2 \lambda \sigma^{6}}{N^{3}} \int \mathrm{d} \mathbf{t} \sum_{a, b=1}^{n} \operatorname{Tr}\left(\bar{\psi}_{b}^{4} \cdot \psi_{a}^{4}\right)-\frac{\lambda \sigma^{8}}{4 N^{9}} \sum_{c=1}^{4} \int \mathrm{d} \mathbf{t} \mathrm{d} \mathbf{t}^{\prime} \sum_{a, b, p, q=1}^{n} \operatorname{Tr}\left[\left(\bar{\psi}_{b}^{4} \cdot \psi_{a}^{4}\right)_{c}\left(\bar{\psi}_{p}^{4} \cdot \psi_{q}^{4}\right)_{c}\right)\right]\right)
\end{aligned}
$$

This leads to the following expression for the effective action 


$$
\begin{aligned}
\mathcal{S}_{\text {eff }}[\psi, \bar{\psi}]= & \int \mathrm{d} t \sum_{a=1}^{n} \sum_{f=1}^{4} \sum_{i} \bar{\psi}_{i}^{f, a} \frac{\mathrm{d}}{\mathrm{d} t} \psi_{i}^{f, a}-\frac{\sigma^{2}-2 \lambda \sigma^{6}}{N^{3}} \int \mathrm{d} \mathbf{t} \sum_{a, b=1}^{n} \operatorname{Tr}\left(\bar{\psi}_{b}^{4} \cdot \psi_{a}^{4}\right) \\
& \left.+\frac{\lambda \sigma^{8}}{4 N^{9}} \sum_{c=1}^{4} \int \mathrm{d} \mathbf{t} \mathrm{d} \mathbf{t}^{\prime} \sum_{a, b, p, q=1}^{n} \operatorname{Tr}\left[\left(\bar{\psi}_{b}^{4} \cdot \psi_{a}^{4}\right)_{c}\left(\bar{\psi}_{p}^{4} \cdot \psi_{q}^{4}\right)_{c}\right)\right],
\end{aligned}
$$

with $\mathrm{dt}^{\prime}=\mathrm{d} t_{3} \mathrm{~d} t_{4}$. We can directly see the effect of the non-Gaussian perturbation on the effective action. Taking $\lambda=0$, we recover the action of the model without quartic perturbation in the disorder. We then define the bilocal fields

$$
\mathrm{G}_{f}^{a b}\left(t_{1}, t_{2}\right)=\frac{1}{N} \sum_{i} \psi_{i}^{f, b}\left(t_{1}\right) \bar{\psi}_{i}^{f, a}\left(t_{2}\right)
$$

and introduce the Lagrange multipliers $\Sigma_{f}^{a b}\left(t_{1}, t_{2}\right)$

$$
\int \mathcal{D} \Sigma_{f}^{a b} \exp \left(-N \int \mathrm{d} \mathbf{t} \sum_{a, b=1}^{n} \sum_{f=1}^{4} \Sigma_{f}^{a b}(\mathbf{t})\left(\mathrm{G}_{f}^{a b}(\mathbf{t})-\frac{1}{N} \sum_{i} \psi_{i}^{f, b}\left(t_{1}\right) \bar{\psi}_{i}^{f, a}\left(t_{2}\right)\right)\right) .
$$

The effective action (41) rewrites as

$$
\begin{aligned}
\mathcal{S}_{\mathrm{eff}}[\psi, \bar{\psi}, \mathrm{G}, \Sigma]= & \int \mathrm{d} \mathbf{t} \sum_{a, b=1}^{n} \sum_{f=1}^{4} \sum_{i} \bar{\psi}_{i}^{f, a}\left(\delta_{a b} \delta\left(t_{1}-t_{2}\right) \partial_{t}-\Sigma_{f}^{a b}(\mathbf{t})\right) \psi_{i}^{f, a}-N\left(\sigma^{2}-2 \lambda \sigma^{6}\right) \int \mathrm{d} \mathbf{t} \sum_{a, b=1}^{n} \prod_{f=1}^{4} \mathrm{G}_{f}^{a b}(\mathbf{t}) \\
& +N \sum_{a, b=1}^{n} \int \mathrm{d} \mathbf{t} \sum_{f=1}^{4} \Sigma_{f}^{a b}(\mathbf{t}) \mathrm{G}_{f}^{a b}(\mathbf{t})+\frac{\lambda \sigma^{8}}{4 N} \int \mathrm{d} \mathbf{t} \mathrm{d} \mathbf{t}^{\prime} \sum_{a, b, p, q=1}^{n} \sum_{c=1}^{4} \mathrm{G}_{c}^{a p}\left(t_{1}, t_{4}\right) \mathrm{G}_{c}^{q b}\left(t_{3}, t_{2}\right) \prod_{f \neq c} \mathrm{G}_{f}^{a b}(\mathbf{t}) \mathrm{G}_{f}^{q p}\left(\mathbf{t}^{\prime}\right) .
\end{aligned}
$$

We now perform the Gaussian integral on the fermionic fields

$$
\begin{aligned}
\left\langle\mathrm{Z}^{n}\right\rangle_{J}= & \int \mathcal{D} \psi_{i}^{f, a} \mathcal{D} \bar{\psi}_{i}^{f, a} \mathcal{D} \mathrm{G}_{f}^{a b} \mathcal{D} \Sigma_{f}^{a b} \exp \left(-\mathcal{S}_{\mathrm{eff}}[\psi, \bar{\psi}, \mathrm{G}, \Sigma]\right) \\
= & \int \mathcal{D} \mathrm{G}_{f}^{a b} \mathcal{D} \Sigma_{f}^{a b} \exp \left(\frac{N}{2} \sum_{a, b=1}^{n} \sum_{f=1}^{4} \log \operatorname{det}\left(\delta_{a b} \delta\left(t_{1}-t_{2}\right) \partial_{t}-\Sigma_{f}^{a b}\left(t_{1}, t_{2}\right)\right)+N\left(\sigma^{2}-2 \lambda \sigma^{6}\right) \int \mathrm{d} \mathbf{t} \sum_{a, b=1}^{n} \prod_{f=1}^{4} \mathrm{G}_{f}^{a b}(\mathbf{t})\right. \\
& \left.-N \sum_{a, b=1}^{n} \int \mathrm{d} \mathbf{t} \sum_{f=1}^{4} \Sigma_{f}^{a b}(\mathbf{t}) \mathrm{G}_{f}^{a b}(\mathbf{t})-\frac{\lambda \sigma^{8}}{4 N} \int \mathrm{d} \mathbf{t} \mathrm{d} \mathbf{t}^{\prime} \sum_{a, b, p, q=1}^{n} \sum_{c=1}^{4} \mathrm{G}_{c}^{a p}\left(t_{1}, t_{4}\right) \mathrm{G}_{c}^{q b}\left(t_{3}, t_{2}\right) \prod_{f \neq c} \mathrm{G}_{f}^{a b}(\mathbf{t}) \mathrm{G}_{f}^{q p}\left(\mathbf{t}^{\prime}\right)\right)
\end{aligned}
$$

We then assume a symmetric saddle point for the replicas and get the effective action

$$
\begin{aligned}
\frac{\mathcal{S}_{\mathrm{eff}}[\mathrm{G}, \Sigma]}{N}= & -\sum_{f=1}^{4} \log \operatorname{det}\left(\delta\left(t_{1}-t_{2}\right) \partial_{t}-\Sigma_{f}\left(t_{1}, t_{2}\right)\right)-\left(\sigma^{2}-2 \lambda \sigma^{6}\right) \int \mathrm{d} \mathbf{t} \prod_{f=1}^{4} \mathrm{G}_{f}(\mathbf{t}) \\
& +\int \mathrm{d} \mathbf{t} \sum_{f=1}^{4} \Sigma_{f}(\mathbf{t}) \mathrm{G}_{f}(\mathbf{t})-\frac{\lambda \sigma^{8}}{4 N^{2}} \int \mathrm{d} \mathbf{t} \mathrm{d} \mathbf{t}^{\prime} \sum_{c=1}^{4} \mathrm{G}_{c}\left(t_{1}, t_{4}\right) \mathrm{G}_{c}\left(t_{3}, t_{2}\right) \prod_{f \neq c} \mathrm{G}_{f}(\mathbf{t}) \mathrm{G}_{f}\left(\mathbf{t}^{\prime}\right) .
\end{aligned}
$$

Let us emphasize that the effective action formula above implies that the variance of the Gaussian disorder is modified by the non-Gaussian perturbation

$$
\sigma^{2} \rightarrow \sigma^{2}-2 \lambda \sigma^{6}
$$

Moreover, let us notice that every term in (46) is of order 1, except for the last term, which is of order $\frac{1}{N^{2}}$, as expected from the universality result. This term can be represented graphically as in Fig. 5.

\section{GROSS-ROSENHAUS SYK MODEL WITH NON-GAUSSIAN DISORDER}

In this section, we follow the steps of the calculation of the previous section and we compute the modification of 
the covariance and of the effective action for a quartic melonic perturbation of the disorder of the GrossRosenhaus SYK model.

As already mentioned in the Introduction, the GrossRosenhaus model [8] is a generalization of the SYK model containing $f$ flavors of fermions, with $N_{a}$ fermions of flavor $a$, appearing $q_{a}$ times in the interaction, so that $q=\sum_{a=1}^{f} q_{a}$. The complex model treated in the previous section can thus be seen as a particular case of a complex version of the Gross-Rosenhaus model treated in this section.

The action of the model writes

$$
\mathcal{S}=\int \mathrm{d} t\left(\sum_{a=1}^{f} \sum_{i=1}^{N_{a}} \psi_{i}^{a} \frac{\mathrm{d}}{\mathrm{d} t} \psi_{i}^{a}+\frac{(i)^{\frac{q}{2}}}{\prod_{a=1}^{f} q_{a} !} \sum_{I} J_{I} \prod_{a=1}^{f} \prod_{p=1}^{q_{a}}\left(\psi_{i_{p}^{a}}^{a}\right)\right),
$$

where $I=i_{1}^{1}, \ldots, i_{q_{1}}^{1}, \ldots, i_{1}^{f}, \ldots, i_{q_{f}}^{f}$. The coupling tensor $J$ is now antisymmetric under permutations of indices in the same family of flavors, with the probability distribution

$$
P(J)=C \exp \left(-\frac{1}{2 \sigma^{2} N} \prod_{a} \frac{N_{a}^{q_{a}}}{\left(q_{a}-1\right) !} \sum_{I} J_{I}^{2}-\frac{\lambda}{4} \frac{\prod_{a} N_{a}^{q_{a}}}{N} \sum_{c=1}^{q} \sum_{I, K} J_{I} J_{I_{\hat{c}} k_{c}} J_{K} J_{K_{\hat{c}} i_{c}}\right),
$$

where $N=\sum_{a} N_{a}$ and $\sigma^{2}=\prod_{a} q_{a} ! \tilde{\sigma}^{2}$, with $\tilde{\sigma}^{2}$ as the variance of the Gaussian distribution. We use again the intermediate field

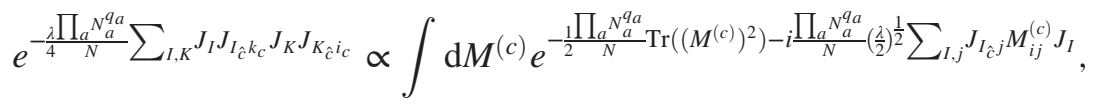

where $M^{(c)}$ is a symmetric real $N_{c} \times N_{c}$ matrix. Using the replica trick and keeping the terms in $J$ we have

$$
\begin{aligned}
& \int \mathcal{D} J \exp \left(-\prod_{a} \frac{N_{a}^{q_{a}}}{\left(q_{a}-1\right) !} \sum_{I} \frac{J_{I}^{2}}{2 \sigma^{2} N}-i \frac{\prod_{a} N_{a}^{q_{a}}}{N}\left(\frac{\lambda}{2}\right)^{\frac{1}{2}} \sum_{c=1}^{q} \sum_{I, j} J_{I_{\hat{c}} j} M_{i j}^{(c)} J_{I}\right. \\
& \left.-\frac{(i)^{\frac{q}{2}}}{\prod_{a=1}^{f} q_{a} !} \int \mathrm{d} t \sum_{r=1}^{n} \sum_{I} J_{I} \prod_{a=1}^{f} \prod_{p=1}^{q_{a}}\left(\psi_{i_{p}^{a}}^{a, r}\right)\right) .
\end{aligned}
$$

As above, let $\mathcal{M}_{c}=\mathbb{1}^{\otimes(c-1)} \otimes M^{(c)} \otimes \mathbb{1}^{\otimes(q-c)}$, where $\mathbb{1}$ is implicitly the identity $N_{a} \times N_{a}$ matrix, for $a=1, \ldots, f$. One then has

$$
\begin{aligned}
& \int \mathcal{D} J \exp \left(-\frac{\prod_{a} N_{a}^{q_{a}}}{2 N} J\left(\frac{1}{\sigma^{2}} \prod_{a=1}^{f} \frac{1}{\left(q_{a}-1\right) !} \mathbb{1}^{\otimes q}+i(2 \lambda)^{\frac{1}{2}} \sum_{c=1}^{q} \mathcal{M}_{c}\right) J-\frac{(i)^{\frac{q}{2}}}{\prod_{a=1}^{f} q_{a} !} \int \mathrm{d} t \sum_{r=1}^{n} \sum_{I} J_{I} \prod_{a=1}^{f} \prod_{p=1}^{q_{a}}\left(\psi_{i_{p}^{a}}^{a, r}\right)\right) \\
& \propto\left(\operatorname{det}\left(\frac{1}{\sigma^{2}} \prod_{a=1}^{f} \frac{1}{\left(q_{a}-1\right) !} \mathbb{1}^{\otimes q}+i(2 \lambda)^{\frac{1}{2}} \sum_{c=1}^{q} \mathcal{M}_{c}\right)\right)^{-\frac{1}{2}} \\
& \quad \times \exp \left(\frac{i^{q} N}{2 \prod_{a=1}^{f} N_{a}^{q_{a}}\left(q_{a} !\right)^{2}} \int \mathrm{d} t_{1} \mathrm{~d} t_{2} \sum_{r, s=1}^{n} \prod_{a=1}^{f} \prod_{p=1}^{q_{a}}\left(\psi_{p}^{a, r}\right)\left(\frac{1}{\sigma^{2}} \prod_{a=1}^{f} \frac{1}{\left(q_{a}-1\right) !} \mathbb{1}^{\otimes q}+i(2 \lambda)^{\frac{1}{2}} \sum_{c=1}^{q} \mathcal{M}_{c}\right)^{-1}\left(\psi_{p}^{a, s}\right)\right) .
\end{aligned}
$$

The replicated generating functional thus writes

$$
\begin{aligned}
\left\langle\mathrm{Z}^{n}\right\rangle_{J}= & \int \mathcal{D} \psi_{i}^{f, a} \mathcal{D} M^{(c)} \exp \left[-\int \mathrm{d} t \sum_{r=1}^{n} \sum_{a=1}^{f} \sum_{i=1}^{N_{a}} \psi_{i}^{a, r} \frac{\mathrm{d}}{\mathrm{d} t} \psi_{i}^{a, r}\right. \\
& -\frac{1}{2} \operatorname{Tr} \log \left(\frac{1}{\sigma^{2}} \prod_{a=1}^{f} \frac{1}{\left(q_{a}-1\right) !} \mathbb{1}^{\otimes q}+i(2 \lambda)^{\frac{1}{2}} \sum_{c=1}^{q} \mathcal{M}_{c}\right)-\frac{1}{2} \frac{\prod_{a} N_{a}^{q_{a}}}{N} \sum_{c=1}^{q} \operatorname{Tr}\left(\left(M^{(c)}\right)^{2}\right) \\
& \left.+\frac{i^{q} N}{2 \prod_{a=1}^{f} N_{a}^{q_{a}}\left(q_{a} !\right)^{2}} \int \mathrm{d} t_{1} \mathrm{~d} t_{2} \sum_{r, s=1}^{n} \prod_{a=1}^{f} \prod_{p=1}^{q_{a}}\left(\psi_{p}^{a, r}\right)\left(\frac{1}{\sigma^{2}} \prod_{a=1}^{f} \frac{1}{\left(q_{a}-1\right) !} \mathbb{1}^{\otimes q}+i(2 \lambda)^{\frac{1}{2}} \sum_{c=1}^{q} \mathcal{M}_{c}\right)^{-1}\left(\psi_{p}^{a, s}\right)\right] .
\end{aligned}
$$


As above, we now write the following series

$$
\begin{aligned}
& \left(\frac{1}{\sigma^{2}} \prod_{a=1}^{f} \frac{1}{\left(q_{a}-1\right) !} \rrbracket^{\otimes q}+i(2 \lambda)^{\frac{1}{2}} \sum_{c=1}^{q} \mathcal{M}_{c}\right)^{-1} \\
& =\sigma^{2} \prod_{a=1}^{f}\left(q_{a}-1\right) ! \mathbb{1}^{\otimes q}+\sigma^{2} \prod_{a=1}^{f}\left(q_{a}-1\right) ! \sum_{k \geq 1}\left(-i \sigma^{2}(2 \lambda)^{\frac{1}{2}} \prod_{a=1}^{f}\left(q_{a}-1\right) !\right)^{k} \sum_{\sum_{i}^{q} k_{i}=k}\left(\begin{array}{c}
k \\
k_{1}, \ldots, k_{q}
\end{array}\right) \prod_{c=1}^{q} \mathcal{M}_{c}^{k_{c}}, \\
& \log \left(\frac{1}{\sigma^{2}} \prod_{a=1}^{f} \frac{1}{\left(q_{a}-1\right) !} \rrbracket^{\otimes q}+i(2 \lambda)^{\frac{1}{2}} \sum_{c=1}^{q} \mathcal{M}_{c}\right) \\
& =-2 \log \left(\sigma \mathbb{1}^{\otimes q}\right)-\sum_{a=1}^{f} \log \left(\left(q_{a}-1\right) ! \rrbracket^{\otimes q}\right)+\log \left(\rrbracket^{\otimes q}+i(2 \lambda)^{\frac{1}{2}} \sigma^{2} \prod_{a=1}^{f}\left(q_{a}-1\right) ! \sum_{c=1}^{q} \mathcal{M}_{c}\right) \\
& =-2 \log \left(\sigma \rrbracket^{\otimes q}\right)-\sum_{a=1}^{f} \log \left(\left(q_{a}-1\right) ! \rrbracket \otimes q\right)-\sum_{k \geq 1} \frac{\left(-i(2 \lambda)^{\frac{1}{2}} \sigma^{2} \prod_{a=1}^{f}\left(q_{a}-1\right) !\right)^{k}}{k} \sum_{\sum_{i}^{q} k_{i}=k}\left(k_{1}, \ldots, k_{q}\right) \prod_{c=1}^{q} \mathcal{M}_{c}^{k_{c}}
\end{aligned}
$$

This leads to

$$
\begin{aligned}
& -\frac{1}{2} \frac{\prod_{a} N_{a}^{q_{a}}}{N} \sum_{c=1}^{q} \operatorname{Tr}\left(\left(M^{(c)}\right)^{2}\right)+\frac{1}{2} \sum_{k \geq 1} \frac{\left(-i(2 \lambda)^{\frac{1}{2}} \sigma^{2} \prod_{a=1}^{f}\left(q_{a}-1\right) !\right)^{k}}{k} \sum_{\sum_{i}^{q} k_{i}=k}\left(\begin{array}{c}
k \\
k_{1}, \ldots, k_{q}
\end{array}\right) \operatorname{Tr}\left(\prod_{c=1}^{q} \mathcal{M}_{c}^{k_{c}}\right) \\
& \quad+\frac{i^{q} N \sigma^{2}}{2 \prod_{a=1}^{f} N_{a}^{q_{a}} q_{a}\left(q_{a} !\right)} \sum_{k \geq 1}\left(-i \sigma^{2}(2 \lambda)^{\frac{1}{2}} \prod_{a=1}^{f}\left(q_{a}-1\right) !\right) \sum_{\sum_{i}^{q} k_{i}=k}^{k}\left(\begin{array}{c}
k \\
k_{1}, \ldots, k_{q}
\end{array}\right) \int \mathrm{dt} \operatorname{tr}\left(\left(\psi_{r}^{q} \cdot \psi_{s}^{q}\right) \prod_{c=1}^{q} \mathcal{M}_{c}^{k_{c}}\right),
\end{aligned}
$$

where we used the notation $\left(\psi_{r}^{q} \cdot \psi_{s}^{q}\right)=\sum_{r, s=1}^{n} \bigotimes_{a=1}^{f} \bigotimes_{p=1}^{q_{a}}\left(\psi_{p}^{a, r} \cdot \psi_{p}^{a, s}\right)$ and $\left(\psi_{p}^{a, r} \cdot \psi_{p}^{a, s}\right)_{i j}=\psi_{i_{p}^{a}}^{a, r} \psi_{j_{p}^{a}}^{a, s}$. For the sake of simplicity, we can rewrite this term in the more compact form

$$
-\frac{1}{2} \frac{\prod_{a} N_{a}^{q_{a}}}{N} \sum_{c=1}^{q} \operatorname{Tr}\left(\left(M^{(c)}\right)^{2}\right)+\frac{1}{2} \operatorname{Tr}\left[\sum_{k \geq 1}\left(-i(2 \lambda)^{\frac{1}{2}} \sigma^{2} \prod_{a=1}^{f}\left(q_{a}-1\right) !\right)^{k} \sum_{\sum_{i}^{q} k_{i}=k}\left(\begin{array}{c}
k \\
k_{1}, \ldots, k_{q}
\end{array}\right) \hat{A}_{k} \prod_{c=1}^{q} \mathcal{M}_{c}^{k_{c}}\right]
$$

where we denoted

$$
\hat{A}_{k}=\frac{\mathbb{1}^{\otimes q}}{k}+\frac{i^{q} N \sigma^{2}}{\prod_{a=1}^{f} N_{a}^{q_{a}} q_{a}\left(q_{a} !\right)} \int \mathrm{d} \mathbf{t}\left(\psi_{r}^{q} \cdot \psi_{s}^{q}\right)
$$

Keeping only the terms in $\sqrt{\lambda}$ in (57), we get

$$
-\frac{1}{2} \frac{\prod_{a} N_{a}^{q_{a}}}{N} \sum_{c=1}^{q} \operatorname{Tr}\left(\left(M^{(c)}\right)^{2}\right)-i \sigma^{2} \prod_{a=1}^{f}\left(q_{a}-1\right) ! \sqrt{\frac{\lambda}{2}} \sum_{c=1}^{q} \operatorname{Tr}\left(\hat{A}_{1} \mathcal{M}_{c}\right) .
$$

This allows us to perform the Gaussian integral over the intermediate fields

$$
\begin{aligned}
& \int \prod_{c=1}^{q} \mathrm{~d} M^{(c)} \exp \left(-\frac{1}{2} \frac{\prod_{a} N_{a}^{q_{a}}}{N} \sum_{c=1}^{q} \operatorname{Tr}\left(\left(M^{(c)}\right)^{2}\right)-i \sigma^{2} \prod_{a=1}^{f}\left(q_{a}-1\right) ! \sqrt{\frac{\lambda}{2}} \sum_{c=1}^{q} \operatorname{Tr}\left(\hat{A}_{1} \mathcal{M}_{c}\right)\right) \\
& \propto \exp \left(-\frac{i^{q} \lambda N^{2} \sigma^{6}}{2 \prod_{a=1}^{f} N_{a}^{2 q_{a}} q_{a}^{3}\left(q_{a} !\right)^{-1}} \int \mathrm{d} \mathbf{t} \operatorname{Tr}\left(\psi_{r}^{q} \cdot \psi_{s}^{q}\right)-\frac{(-1)^{q} \lambda N^{3} \sigma^{8}}{4 \prod_{a=1}^{f} N_{a}^{3 q_{a}} q_{a}^{4}} \sum_{c=1}^{f} \sum_{k=1}^{q_{c}} \int \mathrm{d} \mathbf{t} \mathrm{d} \mathbf{t}^{\prime} \operatorname{Tr}\left[\left(\psi_{r}^{q} \cdot \psi_{s}^{q}\right)_{c, k}\left(\psi_{u}^{q} \cdot \psi_{v}^{q}\right)_{c, k}\right]\right),
\end{aligned}
$$

where $\left(\psi_{r}^{q} \cdot \psi_{s}^{q}\right)_{c, k}=\sum_{r, s=1}^{n} \prod_{a \neq c} \prod_{p \neq k} \operatorname{Tr}\left[\left(\psi_{p}^{a, r} \cdot \psi_{p}^{a, s}\right)\right]\left(\psi_{k}^{c, r} \cdot \psi_{k}^{c, s}\right)$. This leads to the following expression for the effective action: 


$$
\begin{aligned}
\mathcal{S}_{\text {eff }}[\psi]= & \int \mathrm{d} t \sum_{a=1}^{f} \sum_{r=1}^{n} \sum_{i=1}^{N_{a}} \psi_{i}^{a, r} \frac{\mathrm{d}}{\mathrm{d} t} \psi_{i}^{a, r}-\frac{i^{q} N}{2 \prod_{a=1}^{f} N_{a}^{q_{a}} q_{a} q_{a} !}\left(\sigma^{2}-\lambda N \sigma^{6} \prod_{a=1}^{f} \frac{\left(q_{a} !\right)^{2}}{q_{a}^{2} N_{a}^{q_{a}}}\right) \int \mathrm{d} \operatorname{tr}\left(\psi_{r}^{q} \cdot \psi_{s}^{q}\right) \\
& +\frac{(-1)^{q} \lambda N^{3} \sigma^{8}}{4 \prod_{a=1}^{f} N_{a}^{3 q_{a}} q_{a}^{4}} \sum_{c=1}^{f} \sum_{k=1}^{q_{c}} \int \mathrm{d} \mathbf{d} \mathrm{d} \mathbf{t}^{\prime} \operatorname{Tr}\left[\left(\psi_{r}^{q} \cdot \psi_{s}^{q}\right)_{c, k}\left(\psi_{u}^{q} \cdot \psi_{v}^{q}\right)_{c, k}\right] .
\end{aligned}
$$

In order to evaluate the fermionic integral in the expression of the replicated generating function, we introduce the bilocal fields

$$
G_{a}^{r s}\left(t_{1}, t_{2}\right)=\frac{1}{N_{a}} \sum_{i=1}^{N_{a}} \psi_{i}^{a, r}\left(t_{1}\right) \psi_{i}^{a, s}\left(t_{2}\right)
$$

and the Lagrange multipliers $\Sigma_{a}^{r s}\left(t_{1}, t_{2}\right)$

$$
\int \mathcal{D} \Sigma_{a}^{r s} \exp \left(-\int \mathrm{d} \mathbf{t} \sum_{r, s=1}^{n} \sum_{a=1}^{f} \frac{N_{a}}{2} \sum_{a}^{r s}\left(t_{1}, t_{2}\right)\left(G_{a}^{r s}\left(t_{1}, t_{2}\right)-\frac{1}{N_{a}} \sum_{i=1}^{N_{a}} \psi_{i}^{a, r} \psi_{i}^{a, s}\right)\right) .
$$

The effective action (61) then writes

$$
\begin{aligned}
\mathcal{S}_{\mathrm{eff}}[\psi, G, \Sigma]= & \int \mathrm{d} \mathbf{t} \sum_{a=1}^{f} \sum_{r, s=1}^{n} \sum_{i=1}^{N_{a}} \psi_{i}^{a, r}\left(\delta_{r s} \delta\left(t_{1}-t_{2}\right) \partial_{t}-\Sigma_{a}^{r s}(\mathbf{t})\right) \psi_{i}^{a, s}+\int \mathrm{d} \mathbf{t} \sum_{r, s=1}^{n} \sum_{a=1}^{f} \frac{N_{a}}{2} \sum_{a}^{r s}(\mathbf{t}) G_{a}^{r s}(\mathbf{t}) \\
& -\frac{i^{q} N}{2 \prod_{a=1}^{f} q_{a} q_{a} !}\left(\sigma^{2}-\lambda N \sigma^{6} \prod_{a=1}^{f} \frac{\left(q_{a} !\right)^{2}}{q_{a}^{2} N_{a}^{q_{a}}}\right) \int \mathrm{d} \mathbf{t} \sum_{r, s=1}^{n} \prod_{a=1}^{f}\left(G_{a}^{r s}(\mathbf{t})\right)^{q_{a}}+\frac{(-1)^{q} \lambda N^{3} \sigma^{8}}{4 \prod_{a=1}^{f} N_{a}^{q_{a}} q_{a}^{4}} \int \mathrm{d} \mathbf{t} \mathrm{d} \mathbf{t}^{\prime} \\
& \times \sum_{r, s, u, v=1}^{n} \sum_{c=1}^{f} q_{c} G_{c}^{s u}\left(t_{2}, t_{3}\right) G_{c}^{r v}\left(t_{1}, t_{4}\right)\left(G_{c}^{r s}(\mathbf{t})\right)^{q_{c}-1}\left(G_{c}^{u v}\left(\mathbf{t}^{\prime}\right)\right)^{q_{c}-1} \prod_{a \neq c}\left(G_{a}^{r s}(\mathbf{t})\right)^{q_{a}}\left(G_{a}^{u v}\left(\mathbf{t}^{\prime}\right)\right)^{q_{a}}
\end{aligned}
$$

This allows us to perform the Gaussian integral on the fermionic fields

$$
\begin{aligned}
\left\langle\mathrm{Z}^{n}\right\rangle_{J}= & \int \mathcal{D} \psi \mathcal{D} G \mathcal{D} \Sigma e^{-\mathcal{S}_{\text {eff }}[\psi, G, \Sigma]} \\
= & \int \mathcal{D} \psi \mathcal{D} G \mathcal{D} \Sigma \exp \left(-\int \mathrm{d} \mathbf{t} \sum_{a=1}^{f} \sum_{r, s=1}^{n} \frac{N_{a}}{2} \log \operatorname{det}\left(\delta_{r s} \delta\left(t_{1}-t_{2}\right) \partial_{t}-\Sigma_{a}^{r s}(\mathbf{t})\right)\right. \\
& -\int \mathrm{d} \mathbf{t} \sum_{r, s=1}^{n} \sum_{a=1}^{f} \frac{N_{a}}{2} \Sigma_{a}^{r s}(\mathbf{t}) G_{a}^{r s}(\mathbf{t})+\frac{i^{q} N}{2 \prod_{a=1}^{f} q_{a} q_{a} !}\left(\sigma^{2}-\lambda N \sigma^{6} \prod_{a=1}^{f} \frac{\left(q_{a} !\right)^{2}}{q_{a}^{2} N_{a}^{q_{a}}}\right) \int \mathrm{d} \mathbf{t} \sum_{r, s=1}^{n} \prod_{a=1}^{f}\left(G_{a}^{r s}(\mathbf{t})\right)^{q_{a}} \\
& \left.-\frac{(-1)^{q} \lambda N^{3} \sigma^{8}}{4 \prod_{a=1}^{f} N_{a}^{q_{a}} q_{a}^{4}} \int \mathrm{d} \mathbf{t} \mathrm{d} \mathbf{t}^{\prime} \sum_{r, s, u, v=1}^{n} \sum_{c=1}^{f} q_{c} G_{c}^{s u}\left(t_{2}, t_{3}\right) G_{c}^{r v}\left(t_{1}, t_{4}\right)\left(G_{c}^{r s}(\mathbf{t})\right)^{q_{c}-1}\left(G_{c}^{u v}\left(\mathbf{t}^{\prime}\right)\right)^{q_{c}-1} \prod_{a \neq c}\left(G_{a}^{r s}(\mathbf{t})\right)^{q_{a}}\left(G_{a}^{u v}\left(\mathbf{t}^{\prime}\right)\right)^{q_{a}}\right) .
\end{aligned}
$$

Assuming a symmetric saddle point for the replicas, we get the following expression for the effective action:

$$
\begin{aligned}
\mathcal{S}_{\text {eff }}[G, \Sigma]= & \int \mathrm{d} \mathbf{t} \sum_{a=1}^{f} \frac{N_{a}}{2} \log \operatorname{det}\left(\delta\left(t_{1}-t_{2}\right) \partial_{t}-\Sigma_{a}(\mathbf{t})\right)+\int \mathrm{d} \mathbf{t} \sum_{a=1}^{f} \frac{N_{a}}{2} \Sigma_{a}(\mathbf{t}) G_{a}(\mathbf{t}) \\
& -\frac{i^{q} N}{2 \prod_{a=1}^{f} q_{a}}\left(\tilde{\sigma}^{2}-\lambda N \tilde{\sigma}^{6} \prod_{a=1}^{f} \frac{\left(q_{a} !\right)^{4}}{q_{a}^{2} N_{a}^{q_{a}}}\right) \int \mathrm{d} \mathbf{t} \prod_{a=1}^{f}\left(G_{a}(\mathbf{t})\right)^{q_{a}}+(-1)^{q} \frac{\lambda}{4} N^{3} \tilde{\sigma}^{8} \prod_{a=1}^{f} \frac{\left(q_{a} !\right)^{4}}{N_{a}^{q_{a}} q_{a}^{4}} \int \mathrm{d} \mathbf{t} \mathrm{d} \mathbf{t}^{\prime} \\
& \times \sum_{c=1}^{f} q_{c} G_{c}\left(t_{2}, t_{3}\right) G_{c}\left(t_{1}, t_{4}\right)\left(G_{c}(\mathbf{t})\right)^{q_{c}-1}\left(G_{c}\left(\mathbf{t}^{\prime}\right)\right)^{q_{c}-1} \prod_{a \neq c}\left(G_{a}(\mathbf{t})\right)^{q_{a}}\left(G_{a}\left(\mathbf{t}^{\prime}\right)\right)^{q_{a}} .
\end{aligned}
$$


Let us emphasize that the Gaussian variance is now modified by the non-Gaussian perturbation

$$
\tilde{\sigma}^{2} \rightarrow \tilde{\sigma}^{2}-\lambda N \tilde{\sigma}^{6} \prod_{a=1}^{f} \frac{\left(q_{a} !\right)^{4}}{q_{a}^{2} N_{a}^{q_{a}}} .
$$

This expression is a generalization of the modification (47) of the Gaussian variance of the complex model treated in the previous sections.

\section{CONCLUDING REMARKS}

In this paper, we have investigated the effects of nonGaussian average over the random couplings $J$ in a complex SYK model, as well as in a (real) SYK generalization proposed by Gross and Rosenhaus. To our knowledge, this is the first study of the effects of the relaxation of the Gaussianity condition in SYK models when no doublescaling limit is taken.

An interesting perspective appears to us to be the investigation of the effects of such a perturbation from Gaussianity in the case of $q=2$ (fermions with a random mass matrix) and in the case of the real SYK model-a first step towards this latter case having been already made in this paper (since the real SYK model is a particular case of the Gross-Rosenhaus model). The main technical complication in this latter case comes from the fact that one has to deal with graphs that are not necessary bipartite- the removal and reconnection of edges of these graphs (which is the main technical ingredient of our approach) being much more involved.
It would thus be interesting to check whether or not in this case also non-Gaussian perturbation leads to a modification of the variance of the Gaussian distributions of the couplings $J$ at leading order in $N$, as we proved to be the case for the complex version of the SYK model studied here.

\section{ACKNOWLEDGMENTS}

The authors warmly acknowledge Stéphane Dartois for various discussions on the Dyson-Schwinger equations. The authors are partially supported by the CNRS Infiniti ModTens grant. A. T. is partially supported by Grant No. PN 09370102.

\section{APPENDIX: DYSON-SCHWINGER EQUATION FOR THE INTERMEDIATE FIELD}

In this Appendix, we construct the Dyson-Schwinger equations for the matrix intermediate field used in the paper. As already announced in the Introduction, our calculations follow the lines of [31]. The following subsection deals with the complex SYK model and the last subsection deals with the real Gross-Rosenhaus SYK generalization.

\section{Complex SYK}

We first perform the following change of variables:

$$
M^{(c)} \rightarrow \alpha \mathbb{1}+\frac{1}{N} M^{(c)} .
$$

The effective action for the intermediate field in Eq. (37) leads to the following expression:

$$
\begin{aligned}
- & \frac{N}{2} \sum_{c=1}^{4} \operatorname{Tr}\left(M^{(c) 2}\right)-\alpha N^{2} \sum_{c=1}^{4} \operatorname{Tr}\left(M^{(c)}\right) \\
& +\operatorname{Tr}\left(\sum_{k \geq 1} \sum_{k_{1}+k_{2}+k_{3}+k_{4}=k} \frac{\left(-i\left(\frac{\lambda}{2}\right)^{\frac{1}{2}} \sigma^{2}\right)^{k}}{k_{1} ! k_{2} ! k_{3} ! k_{4} !}\left((k-1) ! \rrbracket^{\otimes 4}+\frac{\sigma^{2}}{N^{3}} \int \mathrm{d} \mathbf{t} \sum_{a, b=1}^{n}\left(\bar{\psi}_{b}^{4} \cdot \psi_{a}^{4}\right)\right) \prod_{c=1}^{4}\left(\alpha \rrbracket^{\otimes 4}+\frac{1}{N} \mathcal{M}_{c}\right)^{k_{c}}\right) \\
= & -\frac{N}{2} \sum_{c=1}^{4} \operatorname{Tr}\left(M^{(c) 2}\right)-\alpha N^{2} \sum_{c=1}^{4} \operatorname{Tr}\left(M^{(c)}\right) \\
& +\operatorname{Tr}\left(\sum_{k \geq 1} \sum_{k_{1}+k_{2}+k_{3}+k_{4}=k} \frac{\left(-i\left(\frac{\lambda}{2}\right)^{\frac{1}{2}} \sigma^{2} \alpha\right)^{k}}{k_{1} ! k_{2} ! k_{3} ! k_{4} !}\left((k-1) ! \mathbb{1}^{\otimes 4}+\frac{\sigma^{2}}{N^{3}} \int \mathrm{d} \mathbf{t} \sum_{a, b=1}^{n}\left(\bar{\psi}_{b}^{4} \cdot \psi_{a}^{4}\right)\right) \prod_{c=1}^{4} \sum_{p_{c}=0}^{k_{c}}\left(\begin{array}{c}
k_{c} \\
p_{c}
\end{array}\right) \frac{\mathcal{M}_{c}^{p_{c}}}{(\alpha N)^{p_{c}}}\right),
\end{aligned}
$$

where we recall the notation $\mathcal{M}_{c}=\mathbb{1}^{\otimes(c-1)} \otimes M^{(c)} \otimes \mathbb{1}^{\otimes(4-c)}$. Using the expression above of the action, we can now derive the Dyson-Schwinger equations (recall that these equations can be derived by exploiting the fact that the integration of a total derivative is vanishing)

$$
\begin{aligned}
& 0=\sum_{i j} \int \prod_{c=1}^{4} \mathrm{~d} M^{(c)} \frac{\partial}{\partial M_{i j}^{(d)}}\left[( M ^ { ( d ) } ) _ { i j } ^ { q } \operatorname { e x p } \left(-\frac{N}{2} \sum_{c=1}^{4} \operatorname{Tr}\left(M_{c}^{2}\right)-\alpha N^{2} \sum_{c=1}^{4} \operatorname{Tr}\left(M_{c}\right)\right.\right. \\
& \left.\left.+\operatorname{Tr}\left(\sum_{k \geq 1} \sum_{k_{1}+k_{2}+k_{3}+k_{4}=k} \frac{\left(-i\left(\frac{\lambda}{2}\right)^{\frac{1}{2}} \sigma^{2} \alpha\right)^{k}}{k_{1} ! k_{2} ! k_{3} ! k_{4} !}\left((k-1) ! \mathbb{1}^{\otimes 4}+\frac{\sigma^{2}}{N^{3}} \int \mathrm{d} \mathbf{t} \sum_{a, b=1}^{n}\left(\bar{\psi}_{b}^{4} \cdot \psi_{a}^{4}\right)\right) \prod_{c=1}^{4} \sum_{p_{c}=0}^{k_{c}}\left(\begin{array}{c}
k_{c} \\
p_{c}
\end{array}\right) \frac{\mathcal{M}_{c}^{p_{c}}}{(\alpha N)^{p_{c}}}\right)\right)\right] .
\end{aligned}
$$


This leads to

$$
\begin{aligned}
0= & \left\langle\sum_{i=0}^{q-1} \operatorname{Tr}\left(M^{(d) i}\right) \operatorname{Tr}\left(M^{(d) q-i-1}\right)\right\rangle-N\left\langle\operatorname{Tr}\left(M^{(d) q+1}\right)\right\rangle-\alpha N^{2}\left\langle\operatorname{Tr}\left(M^{(d) q}\right)\right\rangle \\
& +\left\langle\operatorname { T r } \left[\sum_{k \geq 1} \sum_{k_{1}+k_{2}+k_{3}+k_{4}=k} \frac{\left(-i\left(\frac{\lambda}{2}\right)^{\frac{1}{2}} \sigma^{2} \alpha\right)^{k}}{k_{1} ! k_{2} ! k_{3} ! k_{4} !}\left((k-1) ! \rrbracket^{\otimes 4}+\frac{\sigma^{2}}{N^{3}} \int \mathrm{d} \mathbf{t} \sum_{a, b=1}^{n}\left(\bar{\psi}_{b}^{4} \cdot \psi_{a}^{4}\right)\right)\right.\right. \\
& \left.\left.\times\left(\prod_{\substack{c=1 \\
c \neq d}}^{4} \sum_{p_{c}=0}^{k_{c}}\left(\begin{array}{c}
k_{c} \\
p_{c}
\end{array}\right) \frac{\mathcal{M}_{c}^{p_{c}}}{(\alpha N)^{p_{c}}} \sum_{p_{d}=1}^{k_{d}}\left(\begin{array}{c}
k_{d} \\
p_{d}
\end{array}\right) \frac{p_{d}}{\alpha N} \frac{\mathcal{M}_{d}^{q+p_{d}-1}}{(\alpha N)^{p_{d}-1}}\right)\right]\right\rangle .
\end{aligned}
$$

Let us now compute in detail the leading order (LO) and the next to leading order (NLO) of the above Dyson-Schwinger equation. Notice that the LO is of order $N^{3}$ and it writes

$$
0=-\alpha\left\langle\operatorname{Tr}\left(M^{(d) q}\right)\right\rangle+\frac{1}{\alpha} \sum_{k \geq 1} \frac{1}{k} \sum_{k_{1}+k_{2}+k_{3}+k_{4}=k}\left(-i\left(\frac{\lambda}{2}\right)^{\frac{1}{2}} \sigma^{2} \alpha\right)^{k} \frac{k ! k_{d}}{k_{1} ! k_{2} ! k_{3} ! k_{4} !}\left\langle\operatorname{Tr}\left(M^{(d) q}\right)\right\rangle .
$$

This LO equation rewrites as

$\alpha^{2}=\sum_{k \geq 1} \frac{1}{k}\left(-i\left(\frac{\lambda}{2}\right)^{\frac{1}{2}} \sigma^{2} \alpha\right)^{k} \sum_{k_{1}+k_{2}+k_{3}+k_{4}=k} \frac{k ! k_{d}}{k_{1} ! k_{2} ! k_{3} ! k_{4} !}$.

Let us now recall the following identity:

$$
\sum_{k_{1}+\cdots+k_{D}=k} \frac{k !}{k_{1} ! \ldots k_{D} !} \prod_{i=1}^{D} x_{i}^{k_{i}}=\left(x_{1}+\cdots+x_{D}\right)^{k} .
$$

We now derive the above identity with respect to $x_{d}$; this leads to

$\sum_{k_{1}+\cdots+k_{D}=k} \frac{k ! k_{d}}{k_{1} ! \ldots k_{D} !} \prod_{i \neq d}^{D} x_{i}^{k_{i}} x_{d}^{k_{d}-1}=k\left(x_{1}+\cdots+x_{D}\right)^{k-1}$.

Setting all the $x_{i}$ 's equal to 1 , we have

$$
k D^{k-1}=\sum_{k_{1}+\cdots+k_{D}=k} \frac{k ! k_{d}}{k_{1} ! \ldots k_{D} !} .
$$

Using the above result for $D=4$, the $\mathrm{LO}$ equation reduces to

$$
\alpha^{2}=\sum_{k \geq 1}\left(-i\left(\frac{\lambda}{2}\right)^{\frac{1}{2}} \sigma^{2} \alpha\right)^{k} 4^{k-1}=\frac{-i \alpha \sigma^{2} \sqrt{\lambda / 2}}{1+4 i \alpha \sigma^{2} \sqrt{\lambda / 2}}
$$

Finally, we can solve the LO of the Dyson-Schwinger equation and find the values of $\alpha$,

$$
\alpha_{ \pm}=\frac{-1 \pm \sqrt{1+8 \sigma^{4} \lambda}}{8 i \sigma^{2} \sqrt{\lambda / 2}} .
$$

Notice that these are the same values of $\alpha_{ \pm}$found in [31] through a careful use of the saddle point method.

Let us now evaluate the NLO of the Dyson-Schwinger equation. Collecting the terms of order $N^{2}$ in the DysonSchwinger equation (A4), we get

$$
\begin{aligned}
0= & \left\langle\sum_{i=0}^{q-1} \operatorname{Tr}\left(M^{(d) i}\right) \operatorname{Tr}\left(M^{(d) q-i-1}\right)\right\rangle-N\left\langle\operatorname{Tr}\left(M^{(d) q+1}\right)\right\rangle \\
& +\frac{1}{\alpha^{2}}\left\langle\sum_{k \geq 1} \frac{1}{k_{k_{1}+k_{2}+k_{3}+k_{4}=k}}\left(-i\left(\frac{\lambda}{2}\right)^{\frac{1}{2}} \sigma^{2} \alpha\right)^{k} \frac{k !}{k_{1} ! k_{2} ! k_{3} ! k_{4} !} \sum_{c=1}^{4} k_{d}\left(k_{c}-\delta_{c d}\right) \operatorname{Tr}\left(M^{(c) 1-\delta_{c d}}\right) \operatorname{Tr}\left(M^{(d) q+\delta_{c d}}\right)\right\rangle .
\end{aligned}
$$

In order to solve the NLO equation, we first have to evaluate the third term on the rhs of the equation above. In order to do this, we derive Eq. (A8) with respect to $x_{c}$, and we sum over all flavors $c$. This leads to

$$
\sum_{c=1}^{D} \frac{d}{d x_{c}} k\left(x_{1}+\cdots+x_{D}\right)^{k-1}=D k(k-1)\left(x_{1}+\cdots+x_{D}\right)^{k-2}=\sum_{c=1}^{D} \sum_{k_{1}+\cdots+k_{D}=k} \frac{k ! k_{d}\left(k_{c}-\delta_{c d}\right)}{k_{1} ! \ldots k_{D} !} \prod_{i \neq c, d}^{D} x_{i}^{k_{i}} x^{k_{c}-\delta_{c d}} x_{d}^{k_{d}-1-\delta_{c d}} \text {. }
$$


As above, let us set all the $x_{i}$ 's equal to 1 and insert the resulting identity for $D=4$ in the NLO Dyson-Schwinger equation (A12). We get

$$
\begin{aligned}
0= & \left\langle\sum_{i=0}^{q-1} \operatorname{Tr}\left(M^{(d) i}\right) \operatorname{Tr}\left(M^{(d) q-i-1}\right)\right\rangle-N\left\langle\operatorname{Tr}\left(M^{(d) q+1}\right)\right\rangle \\
& +\frac{1}{\alpha^{2}} \sum_{k \geq 1}\left(-i\left(\frac{\lambda}{2}\right)^{\frac{1}{2}} \sigma^{2} \alpha\right)^{k}(k-1) 4^{k-1}\left\langle\sum_{c=1}^{4} \operatorname{Tr}\left(M^{(c) 1-\delta_{c d}}\right) \operatorname{Tr}\left(M^{(d) q+\delta_{c d}}\right)\right\rangle .
\end{aligned}
$$

Using Eq. (A11) the NLO term of the Dyson-Schwinger equation reduces to

$$
0=\left\langle\sum_{i=0}^{q-1} \operatorname{Tr}\left(M^{(d) i}\right) \operatorname{Tr}\left(M^{(d) q-i-1}\right)\right\rangle+\left(\alpha^{2}-1\right) N\left\langle\operatorname{Tr}\left(M^{(d) q+1}\right)\right\rangle+\alpha^{2}\left\langle\sum_{\substack{c=1 \\ c \neq d}}^{4} \operatorname{Tr}\left(M^{(c)}\right) \operatorname{Tr}\left(M^{(d) q}\right)\right\rangle .
$$

Notice that, also in the NLO term of the Dyson-Schwinger equation, we recovered exactly the same result of [31]. Even if we considered a non-Gaussian distribution (a Gaussian term plus a quartic pillow term potential), the first orders of the Dyson-Schwinger equation are the same as in the Gaussian case.

\section{Gross-Rosenhaus SYK generalization}

Let us first consider the formula (57) for the field $M^{(c)}$ and perform the following change of variables:

$$
M^{(c)} \rightarrow \alpha \mathbb{1}+\frac{N}{\prod_{a=1}^{f} N_{a}^{\frac{q_{a}}{2}}} M^{(c)} .
$$

The action (57) thus rewrites

$$
\begin{aligned}
\mathcal{S}\left[M^{(c)}\right]= & \left.\frac{1}{2} N \sum_{c=1}^{q} \operatorname{Tr}\left(\left(M^{(c)}\right)^{2}\right)+\alpha \prod_{a=1}^{f} N_{a}^{\frac{q_{a}}{2}} \sum_{c=1}^{q} \operatorname{Tr}\left(M^{(c)}\right)\right) \\
& -\frac{1}{2} \operatorname{Tr}\left[\sum_{k \geq 1}\left(-i \alpha \sqrt{2 \lambda} \sigma^{2} \prod_{a=1}^{f}\left(q_{a}-1\right) !\right) \sum_{\sum_{i}^{q} k_{i}=k}\left(\begin{array}{c}
k \\
k_{1}, \ldots, k_{q}
\end{array}\right) \hat{A}_{k} \prod_{c=1}^{q} \sum_{p_{c}=0}^{k_{c}}\left(\begin{array}{c}
k_{c} \\
p_{c}
\end{array}\right)\left(\frac{N}{\alpha \prod_{a=1}^{f} N_{a}^{\frac{q_{a}}{2}}}\right)^{p_{c}} \mathcal{M}_{c}^{p_{c}}\right] .
\end{aligned}
$$

The Dyson-Schwinger equation for the intermediate field writes

$$
0=\sum_{i j} \int \prod_{c=1}^{q} \mathrm{~d} M^{(c)} \frac{\partial}{\partial M_{i j}^{(d)}}\left[\left(M^{(d)}\right)_{i j}^{h} e^{-\mathcal{S}[M]}\right] .
$$

This leads to

$$
\begin{aligned}
0= & \left\langle\sum_{i=0}^{h-1} \operatorname{Tr}\left(M^{(d) i}\right) \operatorname{Tr}\left(M^{(d) h-i-1}\right)\right\rangle-N\left\langle\operatorname{Tr}\left(M^{(d) h+1}\right)\right\rangle-\alpha \prod_{a=1}^{f} N_{a}^{\frac{q_{a}}{2}}\left\langle\operatorname{Tr}\left(M^{(d) h}\right)\right\rangle \\
& +\left\langle\operatorname { T r } \left[\sum_{k \geq 1}\left(-i \alpha \sqrt{2 \lambda} \sigma^{2} \prod_{a=1}^{f}\left(q_{a}-1\right) !\right)^{k} \sum_{\sum_{i}^{q} k_{i}=k}\left(\begin{array}{c}
k \\
k_{1}, \ldots, k_{q}
\end{array}\right) \hat{A}_{k}\right.\right. \\
& \left.\left.\times\left(\prod_{\substack{c=1 \\
c \neq d}}^{q} \sum_{p_{c}=0}^{k_{c}}\left(\begin{array}{c}
k_{c} \\
p_{c}
\end{array}\right)\left(\frac{N}{\alpha \prod_{a=1}^{f} N_{a}^{q_{a}}}\right)^{p_{c}} \mathcal{M}_{c}^{p_{c}} \sum_{p_{d}=1}^{k_{d}}\left(\begin{array}{c}
k_{d} \\
p_{d}
\end{array}\right) p_{d}\left(\frac{N}{\alpha \prod_{a=1}^{f} N_{a}^{\frac{q_{a}}{2}}}\right)^{p_{d}} \mathcal{M}_{d}^{h+p_{d}-1}\right)\right]\right\rangle .
\end{aligned}
$$

Let us define $\kappa_{a}=\frac{N_{a}}{N}$, so that the LO of the Dyson-Schwinger equation in the large $N$ limit is of the order of $N^{\frac{q_{a}+2}{2}}$. The LO contribution thus writes 
$0=-\alpha N^{\frac{q_{a}}{2}+1} \prod_{a=1}^{f} \kappa_{a}^{\frac{q_{a}}{2}}\left\langle\operatorname{Tr}\left(M^{(d) h}\right)\right\rangle+\frac{N^{\frac{q_{a}}{2}+1} \prod_{a=1}^{f} \kappa_{a}^{\frac{q_{a}}{2}}}{\alpha \kappa_{d}} \sum_{k \geq 1} \frac{1}{k} \sum_{\sum_{i}^{q} k_{i}=k}\left(\begin{array}{c}k \\ k_{1}, \ldots, k_{q}\end{array}\right)\left(-i\left(\frac{\lambda}{2}\right)^{\frac{1}{2}} \sigma^{2} \alpha \prod_{a=1}^{f}\left(q_{a}-1\right) !\right)^{k} k_{d}\left\langle\operatorname{Tr}\left(M^{(d) h}\right)\right\rangle$,

which becomes

$$
\alpha^{2}=\frac{1}{\kappa_{d}} \sum_{k \geq 1}\left(-i\left(\frac{\lambda}{2}\right)^{\frac{1}{2}} \sigma^{2} \alpha \prod_{a=1}^{f}\left(q_{a}-1\right) !\right)^{k} q^{k-1}=\frac{1}{\kappa_{d}} \frac{-i \alpha \sigma^{2} \sqrt{\lambda / 2} \prod_{a=1}^{f}\left(q_{a}-1\right) !}{1+q i \alpha \sigma^{2} \sqrt{\lambda / 2} \prod_{a=1}^{f}\left(q_{a}-1\right) !},
$$

with

$$
\alpha_{ \pm}=\frac{-1 \pm \sqrt{1+2 q \sigma^{4} \frac{\lambda}{\kappa_{d}}\left(\prod_{a=1}^{f}\left(q_{a}-1\right) !\right)^{2}}}{2 i q \sigma^{2} \sqrt{\lambda / 2} \prod_{a=1}^{f}\left(q_{a}-1\right) !} .
$$

We can note that the saddle point is parametrized by $\kappa_{d}$.

The NLO part of the Dyson-Schwinger equations writes

$$
\begin{aligned}
0= & \left\langle\sum_{i=0}^{h-1} \operatorname{Tr}\left(M^{(d) i}\right) \operatorname{Tr}\left(M^{(d) h-i-1}\right)\right\rangle-N\left\langle\operatorname{Tr}\left(M^{(d) h+1}\right)\right\rangle \\
& +\sum_{k \geq 1} \frac{1}{k} \sum_{\sum_{i}^{q} k_{i}=k}\left(\begin{array}{c}
k \\
k_{1}, \ldots, k_{q}
\end{array}\right)\left(-i\left(\frac{\lambda}{2}\right)^{\frac{1}{2}} \sigma^{2} \alpha \prod_{a=1}^{f}\left(q_{a}-1\right) !\right)^{k} \sum_{c=1}^{q} \frac{k_{d}\left(k_{c}-\delta_{c d}\right)}{\kappa_{d} \kappa_{c} \alpha^{2}}\left\langle\operatorname{Tr}\left(M^{(c) 1-\delta_{c d}}\right) \operatorname{Tr}\left(M^{(d) h+\delta_{c d}}\right)\right\rangle .
\end{aligned}
$$

This can be rewritten as

$$
\begin{aligned}
0= & \left\langle\sum_{i=0}^{h-1} \operatorname{Tr}\left(M^{(d) i}\right) \operatorname{Tr}\left(M^{(d) h-i-1}\right)\right\rangle-N\left\langle\operatorname{Tr}\left(M^{(d) h+1}\right)\right\rangle \\
& +\sum_{k \geq 1}\left(-i\left(\frac{\lambda}{2}\right)^{\frac{1}{2}} \sigma^{2} \alpha \prod_{a=1}^{f}\left(q_{a}-1\right) !\right)^{k}(k-1) q^{k-1} \sum_{c=1}^{q} \frac{1}{\kappa_{d} \kappa_{c} \alpha^{2}}\left\langle\operatorname{Tr}\left(M^{(c) 1-\delta_{c d}}\right) \operatorname{Tr}\left(M^{(d) h+\delta_{c d}}\right)\right\rangle .
\end{aligned}
$$

Finally, we get the following equation:

$$
0=\left\langle\sum_{i=0}^{h-1} \operatorname{Tr}\left(M^{(d) i}\right) \operatorname{Tr}\left(M^{(d) h-i-1}\right)\right\rangle-N\left\langle\operatorname{Tr}\left(M^{(d) h+1}\right)\right\rangle+q \alpha^{2} \sum_{c=1}^{q} \frac{\kappa_{d}}{\kappa_{c}}\left\langle\operatorname{Tr}\left(M^{(c) 1-\delta_{c d}}\right) \operatorname{Tr}\left(M^{(d) h+\delta_{c d}}\right)\right\rangle .
$$

[1] T. A. Brody, J. Flores, J. B. French, P. A. Mello, A. Pandey, and S. S. M. Wong, Random matrix physics: Spectrum and strength fluctuations, Rev. Mod. Phys. 53, 385 (1981).

[2] J. B. French and S. S. M. Wong, Validity of random matrix theories for many-particle systems, Phys. Lett. 33B, 449 (1970).

[3] K. K. Mon and J. B. French, Statistical properties of many particle spectra, Ann. Phys. (N.Y.) 95, 90 (1975).
[4] S. Sachdev and J. Ye, Gapless Spin Fluid Ground State in a Random, Quantum Heisenberg Magnet, Phys. Rev. Lett. 70, 3339 (1993).

[5] O. Parcollet and A. Georges, Non-Fermi-liquid regime of a doped Mott insulator, Phys. Rev. B 59, 5341 (1999).

[6] J. Maldacena and D. Stanford, Comments on the SachdevYe-Kitaev model, Phys. Rev. D 94, 106002 (2016). 
[7] J. Polchinski and V. Rosenhaus, The spectrum in the Sachdev-Ye-Kitaev model, J. High Energy Phys. 04 (2016) 001.

[8] D. J. Gross and V. Rosenhaus, A generalization of SachdevYe-Kitaev, J. High Energy Phys. 02 (2017) 093.

[9] V. Bonzom, L. Lionni, and A. Tanasa, Diagrammatics of a colored SYK model and of an SYK-like tensor model, leading and next-to-leading orders, J. Math. Phys. (N.Y.) 58, 052301 (2017).

[10] V. Bonzom, V. Nador, and A. Tanasa, Diagrammatic proof of the large $N$ melonic dominance in the SYK model, (2018).

[11] S. Carrozza and V. Pozsgay, SYK-like tensor quantum mechanics with $\operatorname{Sp}(N)$ symmetry, Nucl. Phys. B 941, 28 (2019).

[12] G. Sarosi, $\mathrm{AdS}_{2}$ holography and the SYK model, Proc. Sci., Modave2017 (2018) 001.

[13] V. Rosenhaus, An introduction to the SYK model, (2018).

[14] R. Gurau, Random Tensor Models (Oxford University Press, New York, 2017).

[15] R. Gurau, The complete 1/N expansion of a SYK-like tensor model, Nucl. Phys. B916, 386 (2017).

[16] R. Gurau, Quenched equals annealed at leading order in the colored SYK model, Europhys. Lett. 119, 30003 (2017).

[17] S. Dartois, H. Erbin, and S. Mondal, Conformality of $1 / N$ corrections in SYK-like models, (2017).

[18] É. Fusy, L. Lionni, and A. Tanasa, Combinatorial study of graphs arising from the Sachdev-Ye-Kitaev model, (2018).

[19] T. Krajewski and R. Toriumi, Polchinski's exact renormalisation group for tensorial theories: Gaussian universality and power counting, J. Phys. A 49, 385401 (2016).

[20] T. Krajewski and R. Toriumi, Polchinski's equation for group field theory, Fortschr. Phys. 62, 855 (2014).

[21] T. Krajewski and R. Toriumi, Exact renormalisation group equations and loop equations for tensor models, SIGMA 12, 068 (2016).

[22] T. Krajewski and R. Toriumi, Power counting and scaling for tensor models, Proc. Sci., CORFU2015 (2016) 116.
[23] R. Gurau, Universality for random tensors, Ann. Inst. H. Poincaré Probab. Stat. 50, 1474 (2014).

[24] R. Gurau, V. Bonzom, and M. Smerlak, Universality in $p$-spin glasses with correlated disorder, J. Stat. Mech. (2013) L02003.

[25] R. Pascalie, C. I. Pérez-Sánchez, and R. Wulkenhaar, Correlation functions of $\mathrm{U}(N)$-tensor models and their Schwinger-Dyson equations [Ann. Inst. Henri Poincare (to be published)].

[26] R. Pascalie, C. I. Pérez-Sánchez, A. Tanasa, and R. Wulkenhaar, On the large N limit of the Schwinger-Dyson equation of tensor field theory, [J. Math. Phys. (to be published)].

[27] S. Carrozza and A. Tanasa, $O(N)$ Random Tensor Models, Lett. Math. Phys. 106, 1531 (2016).

[28] I. R. Klebanov and G. Tarnopolsky, Uncolored random tensors, melon diagrams, and the Sachdev-Ye-Kitaev models, Phys. Rev. D 95, 046004 (2017).

[29] I. R. Klebanov, F. Popov, and G. Tarnopolsky, TASI lectures on large $N$ tensor models, Proc. Sci., TASI2017 (2018) 004.

[30] M. Berkooz, M. Isachenkov, V. Narovlansky, and G. Torrents, Towards a full solution of the large $\mathrm{N}$ doublescaled SYK model, J. High Energy Phys. 03 (2019) 079.

[31] V. A. Nguyen, S. Dartois, and B. Eynard, An analysis of the intermediate field theory of $\mathrm{T}^{4}$ tensor model, J. High Energy Phys. 01 (2015) 013.

[32] J. Zinn-Justin, Quantum Field Theory and Critical Phenomena, International Series of Monographs on Physics (Oxford Science Publications, 2002).

[33] J. Polchinski, Renormalization and effective Lagrangians, Nucl. Phys. B231, 269 (1984).

[34] V. Bonzom, Revisiting random tensor models at large $N$ via the Schwinger-Dyson equations, J. High Energy Phys. 03 (2013) 160.

[35] S. Dartois, R. Gurau, and V. Rivasseau, Double scaling in tensor models with a quartic interaction, J. High Energy Phys. 09 (2013) 088. 